\title{
A clothoid-based three-dimensional curve for attitude planning
}

\author{
Vicent Girbés*, Gloria Vanegas ${ }^{\dagger}$, and Leopoldo Armesto \\ Universitat Politècnica de València, Valencia, 46022, Spain
}

\section{Introduction}

Interest in flying robots, also known as unmanned aerial vehicles (UAVs), has grown during last years in both military and civil fields [1, 2]. The same happens to autonomous underwater vehicles (AUVs) [3]. These vehicles, UAVs and AUVs, offer a wide variety of possible applications and challenges, such as control, guidance or navigation [2, 3].

In this sense, heading and attitude control in UAVs is very important [4], particularly relevant in airplanes (fixed-wing flying vehicles), because they are strongly non-linear, coupled, and tend to be underactuated systems with non-holonomic constraints. Hence, designing a good attitude controller is a difficult task [5, 6, 7, 8, 9], where stability must be taken into account by the controller [10]. Indeed, if the reference is too demanding for the controller or non-achievable because its dynamics is too fast, the vehicle might become unstable. In order to address this issue, autonomous navigation systems usually include a high-level path planner to generate smooth reference trajectories to be followed by the vehicle using a low-level controller. Usually a set of waypoints is given in GPS coordinates, normally from a map, in order to apply a smooth point-to-point control trajectory [11, 12].

There are plenty of space curves commonly used for UAV and AUV navigation, such as Bézier, B-spline, NURBS or other parametric curves [13, 14, 15, 16, 17]. But none of them are intuitive for attitude planning, because they are very sensitive to parameter initialization (control points and weights) [18]. In addition to this, they do not take vehicle's constraints into account and/or require optimization procedures, which may be not feasible for real-time applications. For all these reasons, these parametric curves (polynomial and rational) are out of the scope of the paper and, therefore, are not used in our experimentation. Another drawback of these types of parametric curves is the absence of physical meaning, and it might be unclear to get the relationship between their design parameters and system's dynamic variables. In other words, vehicle's constraints or control limitations (maximum input/output values) cannot be taken into account $a$ priori in the trajectory generation, because it is difficult or not possible to do so. For that reason, other solutions propose the use of kinematic and dynamic models to generate a trajectory [19, 20, 21, 22], although optimization procedures are required to minimize/maximize functions such as time, distance, curvature, velocity, acceleration, jerk, etc. [23].

In two-dimensional (2D) planning, most authors concatenate straight lines, circular arcs, and some transition curves [24, 25, 26, 27]. Among all proposed 2D curves in the bibliography, clothoids (also known as Euler or Cornu spirals [28]) play an important role, since it is proven to be the shortest path [29, 30]. Besides, clothoids have "nice"

\footnotetext{
*Associate researcher at Instituto de Diseño y Fabricación, vgirbes@idf.upv.es

${ }^{\dagger}$ PhD student at Department of Systems Engineering and Automation, glovaza@doctor.upv.es

†Lecturer at Department of Systems Engineering and Automation, larmesto@idf.upv.es
} 
geometric properties, including a close relation between their scaling parameter and physical phenomena (angular velocity, normal acceleration and jerk). Clothoids have been studied in depth to control non-holonomic ground mobile robots [29, 30, 31, 32], proving that clothoid's parameters have a direct effect in safety and comfort.

In order to guarantee $C^{2}$-continuity in three-dimensional (3D) planning, the easiest and most intuitive approach is setting null curvature and torsion in the joining points, as done in 2D planning [29, 30]. This way, straight segments and turnings (made of transition curves) can be easily concatenated, while guaranteeing curvature and torsion continuity. Thus, borrowing the idea from planar movement, space clothoids can also be used in path-planning and navigation applications. However, even though the benefits of planar clothoids have been proven, researchers have paid little attention to the use of such curves in 3D space. A few works proposed the use of clothoids in path-planning for UAVs [33, 34, 35]. Others instead, approached a similar problem from the fields of road design [36] and computer graphics [37, 38, 39].

Nevertheless, only [37] seems to match the exact definition of a 3D clothoid (C3D), which is a curve whose curvature and torsion evolve linearly with respect to arc-length. But, the problem is that this approach is based on a Frenet-Serret frame [40] and requires both a numerical integration and an optimization procedure [37]. Moreover, it is not possible to know a priori the increment in position and orientation with respect to curve's origin.

In particular, the paper focuses on the problem of attitude planning, where an aircraft flying straight needs to perform a smooth maneuver to change its direction and height, for instance to avoid an imminent collision. In fact, we seek for a curve that can be used in a local planning framework as smooth reference trajectory for obstacle avoidance of fixed-wing planes and other UAVs, as well as for planning more complex maneuvers, combining straight lines and other three-dimensional curves to generate smooth paths in a global planning framework. In this paper we present a novel clothoid-based three-dimensional curve (coined as $\mathrm{Cb} 3 \mathrm{D}$ ), which has an analytical solution, allows real-time computation for most of applications and provides some interesting properties for path-planning problems, such as scalability, symmetry, smoothness, monotonicity, etc. The integration of this curve within either local or global planning algorithms is out of the scope of this paper.

The paper is organized as follows. Section $\Pi$ introduces some preliminaries about differential geometry of space curves, as well as 2D and 3D clothoids. The problem statement is explained in this section too. The proposed Cb3D curve is defined in Section III], where some lemmas and properties are also proved in Appendix A. In order to evaluate the proposed Cb3D curve against a C3D, a comparative analysis is performed in Section $[\mathrm{V}$, with some simulations using FlightGear in Section $\mathrm{V}$. Finally, the paper ends with a discussion of results in Section VI 


\section{Preliminaries}

\section{Space Curves}

Any space curve $\mathbf{C}(s)$, parametrized by its arc-length $s$ in a three-dimensional space $\mathbb{R}^{3}$, is determined by its curvature $\kappa(s)$ and its torsion $\tau(s)$, according to the fundamental theorem of space curves [40]. Intuitively, a curve can be obtained from a straight line by bending (curvature) and twisting (torsion). So, for $|\kappa(s)|>0$ and torsion $\tau(s)$, there exists a unique space curve defined by its Frenet-Serret equations as follows

$$
\left[\begin{array}{c}
\mathbf{T}^{\prime}(s) \\
\mathbf{N}^{\prime}(s) \\
\mathbf{B}^{\prime}(s)
\end{array}\right]=\left[\begin{array}{ccc}
0 & \kappa(s) & 0 \\
-\kappa(s) & 0 & \tau(s) \\
0 & -\tau(s) & 0
\end{array}\right]\left[\begin{array}{l}
\mathbf{T}(s) \\
\mathbf{N}(s) \\
\mathbf{B}(s)
\end{array}\right]
$$

being $\mathbf{T}(s), \mathbf{N}(s)$, and $\mathbf{B}(s)$ the tangent, normal, and binormal vectors of a right-handed unit orthogonal system, respectively. While $\mathbf{T}^{\prime}(s)=d \mathbf{T}(s) / d s, \mathbf{N}^{\prime}(s)=d \mathbf{N}(s) / d s, \mathbf{B}^{\prime}(s)=d \mathbf{B}(s) / d s$ are the first derivatives of such vectors.

Thus, this orthogonal system is defined as $\mathbf{R}(s):=[\mathbf{T}(s) \mathbf{N}(s) \mathbf{B}(s)]$ and it can be integrated from Eq. (1) based on a specific pair of functions of curvature $\kappa(s)$ and torsion $\tau(s)$, with a given initial value $\mathbf{R}(0):=[\mathbf{T}(0) \mathbf{N}(0) \mathbf{B}(0)]$. The position is obtained by integrating the tangent vector:

$$
\mathbf{C}(s):=\mathbf{C}(0)+\int_{0}^{s} \mathbf{T}(\xi) d \xi
$$

On the other hand, based on a curve $\mathbf{C}(s)$, we can obtain the tangent $\mathbf{T}(s)$, normal $\mathbf{N}(s)$, and binormal $\mathbf{B}(s)$ vectors. Hence, taking into account the theory of differential geometry of curves [40], the following equalities for the mobile trihedron of any curve $\mathbf{C}(s)$ are defined

$$
\begin{aligned}
\mathbf{T}(s) & :=\frac{\mathbf{C}^{\prime}(s)}{\left\|\mathbf{C}^{\prime}(s)\right\|} \\
\mathbf{N}(s) & :=\frac{\mathbf{T}^{\prime}(s)}{\left\|\mathbf{T}^{\prime}(s)\right\|}=\frac{\left[\mathbf{C}^{\prime}(s) \times \mathbf{C}^{\prime \prime}(s)\right] \times \mathbf{C}^{\prime}(s)}{\left\|\left[\mathbf{C}^{\prime}(s) \times \mathbf{C}^{\prime \prime}(s)\right] \times \mathbf{C}^{\prime}(s)\right\|} \\
\mathbf{B}(s) & :=\mathbf{T}(s) \times \mathbf{N}(s)=\frac{\mathbf{C}^{\prime}(s) \times \mathbf{C}^{\prime \prime}(s)}{\left\|\mathbf{C}^{\prime}(s) \times \mathbf{C}^{\prime \prime}(s)\right\|}
\end{aligned}
$$

being $\mathbf{C}^{\prime}(s)=d \mathbf{C}(s) / d s, \mathbf{C}^{\prime \prime}(s)=d^{2} \mathbf{C}(s) / d s^{2}, \mathbf{C}^{\prime \prime \prime}(s)=d^{3} \mathbf{C}(s) / d s^{3}$ the derivatives of position vector $\mathbf{C}(s)$.

\section{Planar and spatial clothoids}

An Euler spiral or planar clothoid (C2D) is defined in $\mathbb{R}^{2}$ as the curve whose curvature varies linearly with respect to arc-length

$$
\kappa(s):=\kappa_{0}+\sigma_{\kappa} s
$$


where $\kappa_{0}$ is the initial curvature and $\sigma_{\kappa}:=d \kappa(s) / d s$ is the clothoid's sharpness, which is related to its homothety factor or scale $K$ by $\sigma_{K}:=\pi / K^{2}$, and clothoid's tangent angle is

$$
\beta(s, \mathbf{p}):=\beta_{0}+\kappa_{0} s+\frac{\sigma_{\kappa}}{2} s^{2}
$$

where $\beta_{0}$ is the initial tangent angle and $\mathbf{p}=\left\{\beta_{0}, \kappa_{0}, \sigma_{\kappa}\right\}$ is the parameter vector (for notation compactness). In addition to this, the tangent vector can be expressed as

$$
\mathbf{T}(s, \mathbf{p}):=\left[\begin{array}{c}
\cos (\beta(s, \mathbf{p})) \\
\sin (\beta(s, \mathbf{p}))
\end{array}\right]
$$

From Eqs. [2], 77, and (8), well-known equations for a planar clothoid (contained in plane XY) [28], noted as $\mathbf{C}(s, \mathbf{p})$, can be derived from Fresnel integrals as follows

$$
\mathbf{C}(s, \mathbf{p})=\left[\begin{array}{l}
\mathcal{C}(s, \mathbf{p}) \\
\mathcal{S}(s, \mathbf{p})
\end{array}\right]:=\left[\begin{array}{c}
\int_{0}^{s} \cos \left(\beta_{0}+\kappa_{0} \xi+\frac{\sigma_{K}}{2} \xi^{2}\right) d \xi \\
\int_{0}^{s} \sin \left(\beta_{0}+\kappa_{0} \xi+\frac{\sigma_{K}}{2} \xi^{2}\right) d \xi
\end{array}\right]
$$

being $C(s, \mathbf{p})$ and $\mathcal{S}(s, \mathbf{p})$ the Fresnel's cosine and sine integrals. Note that without loss of generality, the clothoid is assumed to be origin-centered, that is $\mathbf{C}(0)=\mathbf{0}$ in Eq. (2).

It is a well-known fact that clothoids provide some interesting geometric, aspect-appearance, comfort, and safety advantages, which are worth to be used in road, railway, and roller coaster design [41, 42]. Clothoids are also good to control non-holonomic vehicles [30,43, 44]. Unfortunately, there is no closed-form solution to compute Fresnel integrals, although it is still possible to analytically compute some geometric properties such as curvature and tangent angle. Despite of clothoid not having analytical solution, some approximations allow real-time computation [32, 45, 46, 47, 48]. Thus, due to their good accuracy to compute clothoids, these approximations are considered, henceforth, as analytical solution. Hence, there is no loss of generality by assuming that clothoids can be computed analytically with errors up to $10^{-20}$ [46].

The authors of [37] introduced the 3D Euler spiral or clothoid (C3D), defined in $\mathbb{R}^{3}$, as a curve whose curvature varies as in Eq. (6) and its torsion evolves as

$$
\tau(s):=\tau_{0}+\sigma_{\tau} s
$$

where $\tau_{0}$ is the initial torsion, and $\sigma_{\tau}:=d \tau / d s$ its first geometric derivative or torsion sharpness.

The main contribution of [37] is the development of a smooth curve to join two arbitrary point-vector configurations in the space for 3D curve completion. The authors used Frenet-Serret formulas (Eq. (1D) to compute a space curve as 
in Eq. (2). To find a solution to the problem, they implemented an optimization procedure using Gradient-descent algorithm [49] to get the optimum values of initial curvature $\kappa_{0}$ and torsion $\tau_{0}$, as well as sharpness of curvature $\sigma_{\kappa}$ and torsion $\sigma_{\tau}$. Therefore, their solution could take any initial and final values of curvature and torsion.

However, aerial or underwater robots moving on a 3D space cannot follow arbitrary curves with instantaneous changes in orientation, curvature, or torsion. Therefore, in many practical cases, apart from taking into account non-holonomic constraints, trajectory planners use robot's current state to set initial values of curvature and torsion. In these cases, the algorithm in [37] only allows to reach either an arbitrary target position or orientation at a time, but not both simultaneously.

\section{Problem Statement}

As stated in section $\Pi$ it is possible to compute a curve $\mathbf{C}(s)$ using Frenet-Serret matrix, Eqs. (1) and (2). However, the main drawback is that, apart from some particular cases such as straight lines, helices, and some geometric curves, it is not possible to know a priori the position and orientation of the final configuration based on generic curvature and torsion functions. In order to compute them, numerical methods need to be used [49], which have a high computation cost for small integration steps. Besides, accuracy is inversely proportional to the integration step size. In these cases, an optimization procedure is required to solve planning problems to reach final Cartesian position and/or orientation [37], which is highly computationally demanding.

In this sense, it is of particular relevance that the proposed curve can provide not only analytical expressions and closed-form implementation and synthesis (curve design), with low computation time and high accuracy, but also some interesting properties/operations such as scaling, computing symmetric curves, ensuring smoothness, computing orientation, generalizing other simplistic curves, and ensuring that position and orientation increase monotonically [50].

In particular, we seek for a curve parametrized by a generic vector $\mathbf{p}$, noted as $\mathbf{C}(s, \mathbf{p})$. Actually, the parameter vector can be divided into two subsets $\mathbf{p}:=\left[\mathbf{p}_{0} \tilde{\mathbf{p}}\right]$, where $\mathbf{p}_{0}$ is a list of parameters defining initial conditions, and $\tilde{\mathbf{p}}$ is the design parameter vector. The goal is to compute $\tilde{\mathbf{p}}^{*}$ such that the tangent vector of the curve $\mathbf{T}(s, \mathbf{p})$ equals an arbitrary target tangent vector $\mathbf{T}^{*}$ for a given $s>0$.

Equivalently to the problem statement in [37], the problem to solve can be stated as an orientation minimization problem

$$
\tilde{\mathbf{p}}^{*}=\arg _{\tilde{\mathbf{p}}} \min \left\|\mathbf{T}^{*}-\mathbf{T}(s, \mathbf{p})\right\|
$$

Without loss of generality, it can be assumed that the local frame of the curve is coincident to the global coordinate frame, which implies that the start point is $\mathbf{C}(0, \mathbf{p})=\left[\begin{array}{lll}0 & 0 & 0\end{array}\right]^{T}$. 


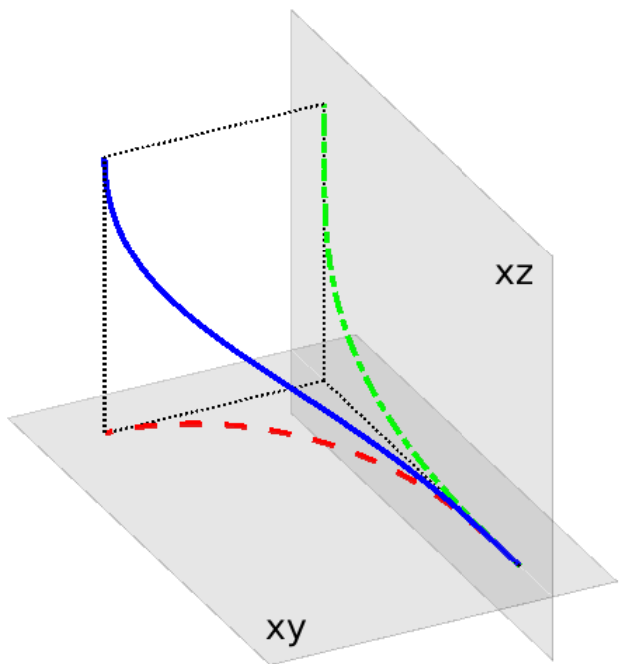

Fig. 1 Cb3D curve (blue) and its projections onto $X Y$ plane (dashed red) and $X Z$ plane dash-dotted green).

\section{Clothoid-based three-dimensional curve}

A clothoid-based three-dimensional curve (Cb3D) is defined as a composition of two planar clothoids. As depicted in Fig. 1, each clothoid is contained in a plane orthogonal to each other (clothoids are defined in planes $X Y$ and $X Z$ ). However, the clothoid contained in plane $X Z$ is indeed a clothoid whose length depends on the clothoid on plane $X Y$, as explained later.

\section{Curve definition and properties}

Lemma III.1 Let $\mathbf{C}_{1}\left(q, \mathbf{p}_{1}\right):=\left[\begin{array}{l}\mathcal{C}\left(q, \mathbf{p}_{1}\right) \\ \mathcal{S}\left(q, \mathbf{p}_{1}\right)\end{array}\right] \in \mathbb{R}^{2}$ be a first planar clothoid contained in plane $X Y$ with arc-length $q$ and parameters $\mathbf{p}_{1}=\left\{\psi_{0}, \epsilon_{0}, \mu\right\}$, and $\mathbf{C}_{2}\left(w, \mathbf{p}_{2}\right):=\left[\begin{array}{l}\mathcal{C}\left(w, \mathbf{p}_{2}\right) \\ \mathcal{S}\left(w, \mathbf{p}_{2}\right)\end{array}\right] \in \mathbb{R}^{2}$ a second planar clothoid contained in plane $X Z$ with arc-length $w$ and parameters $\mathbf{p}_{2}=\left\{\theta_{0}, v_{0}, \rho\right\}$, both defined as in Eq. 99. A Cb3D curve can be computed as a composition of both planar clothoids as follows

$$
\mathbf{C}(s, \mathbf{p}) \equiv \mathbf{C}\left(s, \mathbf{p}_{1}, \mathbf{p}_{2}\right):=\left\{\begin{array}{c}
\mathcal{C}\left(C\left(s, \mathbf{p}_{2}\right), \mathbf{p}_{1}\right) \\
\mathcal{S}\left(C\left(s, \mathbf{p}_{2}\right), \mathbf{p}_{1}\right) \\
-\mathcal{S}\left(s, \mathbf{p}_{2}\right)
\end{array}\right.
$$

where the spatial curve has the following properties/operations:

A Scalability: $\lambda \mathbf{C}\left(s, \mathbf{p}_{1}, \mathbf{p}_{2}\right)=\mathbf{C}\left(\lambda s, \lambda^{-1} \cdot \mathbf{p}_{1}, \lambda^{-1} \cdot \mathbf{p}_{2}\right)$, with $\lambda^{-1}:=\left[1, \frac{1}{\lambda}, \frac{1}{\lambda^{2}}\right]$.

B Symmetry: $\mathbf{C}\left(s, \mathbf{q} \cdot \mathbf{p}_{1}, \mathbf{q} \cdot \mathbf{p}_{2}\right)=-\mathbf{C}\left(-s, \mathbf{q} \cdot \mathbf{p}_{1}, \mathbf{q} \cdot \mathbf{p}_{2}\right)$, with $\mathbf{q}:=[ \pm 1, \mp 1, \pm 1]$. 
C Smoothness: A Cb3D curve is $C^{\infty}$ smooth with $\epsilon_{0} \neq 0$ or $\mu \neq 0 ; v_{0} \neq 0$ or $\rho \neq 0$.

D Orientation: Pitch $\theta\left(s, \mathbf{p}_{2}\right)$, yaw $\psi\left(s, \mathbf{p}_{1}, \mathbf{p}_{2}\right)$, and roll $\phi\left(s, \mathbf{p}_{1}, \mathbf{p}_{2}\right)$ angles defined as

$$
\begin{aligned}
\theta\left(s, \mathbf{p}_{2}\right) & :=\theta_{0}+v_{0} s+\frac{\rho}{2} s^{2} \\
\psi\left(s, \mathbf{p}_{1}, \mathbf{p}_{2}\right) & :=\psi_{0}+\epsilon_{0} C\left(s, \mathbf{p}_{2}\right)+\frac{\mu}{2} C^{2}\left(s, \mathbf{p}_{2}\right) \\
\phi\left(s, \mathbf{p}_{1}, \mathbf{p}_{2}\right) & :=-\arcsin \left(\frac{\theta^{\prime}\left(s, \mathbf{p}_{2}\right)}{\sqrt{\omega\left(s, \mathbf{p}_{1}, \mathbf{p}_{2}\right)^{2}+\theta^{\prime}\left(s, \mathbf{p}_{2}\right)^{2}}}\right)
\end{aligned}
$$

being $\omega\left(s, \mathbf{p}_{1}, \mathbf{p}_{2}\right):=\cos \left(\theta\left(s, \mathbf{p}_{2}\right)\right) \psi^{\prime}\left(s, \mathbf{p}_{1}, \mathbf{p}_{2}\right), \theta^{\prime}\left(s, \mathbf{p}_{2}\right):=d \theta\left(s, \mathbf{p}_{2}\right) / d s$, and $\psi^{\prime}\left(s, \mathbf{p}_{1}, \mathbf{p}_{2}\right):=d \psi\left(s, \mathbf{p}_{1}, \mathbf{p}_{2}\right) / d s$ where tangent, normal, and binormal vectors can be computed as:

$$
\begin{aligned}
& \mathbf{T}\left(s, \mathbf{p}_{1}, \mathbf{p}_{2}\right):=\left[\begin{array}{c}
\cos \left(\psi\left(s, \mathbf{p}_{1}, \mathbf{p}_{2}\right)\right) \cos \left(\theta\left(s, \mathbf{p}_{2}\right)\right) \\
\sin \left(\psi\left(s, \mathbf{p}_{1}, \mathbf{p}_{2}\right)\right) \cos \left(\theta\left(s, \mathbf{p}_{2}\right)\right) \\
-\sin \left(\theta\left(s, \mathbf{p}_{2}\right)\right)
\end{array}\right] \\
& \mathbf{N}\left(s, \mathbf{p}_{1}, \mathbf{p}_{2}\right):=\frac{\mathbf{T}^{\prime}\left(s, \mathbf{p}_{1}, \mathbf{p}_{2}\right)}{\left\|\mathbf{T}^{\prime}\left(s, \mathbf{p}_{1}, \mathbf{p}_{2}\right)\right\|} \\
& \mathbf{B}\left(s, \mathbf{p}_{1}, \mathbf{p}_{2}\right):=\mathbf{T}\left(s, \mathbf{p}_{1}, \mathbf{p}_{2}\right) \times \mathbf{N}\left(s, \mathbf{p}_{1}, \mathbf{p}_{2}\right)
\end{aligned}
$$

E Straight line: A Cb3D curve is a straight line iff $\mathbf{p}_{1}=\left\{\forall \psi_{0}, \epsilon_{0}=0, \mu=0\right\}$ and $\mathbf{p}_{2}=\left\{\forall \theta_{0}, v_{0}=0, \rho=0\right\}$.

F Circular arc: A Cb3D curve is a circular arc iff $\mathbf{p}_{1}=\left\{\forall \psi_{0}, \epsilon_{0} \neq 0, \mu=0\right\}$ and $\mathbf{p}_{2}=\left\{\theta_{0}=0, v_{0}=0, \rho=0\right\}$, or $\mathbf{p}_{1}=\left\{\forall \psi_{0}, \epsilon_{0}=0, \mu=0\right\}$ and $\mathbf{p}_{2}=\left\{\forall \theta_{0}, v_{0} \neq 0, \rho=0\right\}$.

G Planar clothoid: A Cb3D curve is a planar clothoid iff $\mathbf{p}_{1}=\left\{\psi_{0}=0, \epsilon_{0}=0, \mu=0\right\}$ and $\forall \mathbf{p}_{2}$, or $\mathbf{p}_{2}=\left\{\theta_{0}=\right.$ $\left.0, v_{0}=0, \rho=0\right\}$ and $\forall \mathbf{p}_{1}$.

H Circular helix: A Cb3D curve is a circular helix iff $\mathbf{p}_{1}=\left\{\forall \psi_{0}, \epsilon_{0} \neq 0, \mu=0\right\}$ and $\mathbf{p}_{2}=\left\{\theta_{0} \neq 0, v_{0}=0, \rho=0\right\}$.

I Monotonic increasing in position of $\mathbf{C}\left(s, \mathbf{p}_{1}, \mathbf{p}_{2}\right)$ for any $s>0$, with $\theta\left(s, \mathbf{p}_{2}\right) \in[-\pi / 2,0]$ and $\psi\left(s, \mathbf{p}_{1}, \mathbf{p}_{2}\right) \in$ $[0, \pi / 2]$, iff $-\pi / 2 \leq \theta_{0} \leq 0,0 \leq \psi_{0} \leq \pi / 2, v_{0} \leq 0, \epsilon_{0} \geq 0, \rho \leq 0$ and $\mu \geq 0$.

$\mathrm{J}$ Monotonic increasing pitch and yaw angles for any $s>0$, with $\theta\left(s, \mathbf{p}_{2}\right) \in[0, \pi / 2]$ and $\psi\left(s, \mathbf{p}_{1}, \mathbf{p}_{2}\right) \in[0, \pi]$, iff $0 \leq \theta_{0} \leq \pi / 2,0 \leq \psi_{0} \leq \pi, v_{0} \geq 0, \epsilon_{0} \geq 0, \rho \geq 0$ and $\mu \geq 0$.

Proof Appendix A contains a detailed list of proofs for the properties stated in LemmaIII.1.

Fig. 2 shows a Cb3D curve $\mathbf{C}\left(s, \mathbf{p}_{1}, \mathbf{p}_{2}\right)$ with the following parameters $\mathbf{p}_{2}=\{0,0,-\pi\}$ and $\mathbf{p}_{1}=\left\{0,0, \pi / C^{2}\left(1, \mathbf{p}_{2}\right)\right\}$, with arc-length $s \in[0,1] \mathrm{m}$. The final tangent vector is $\mathbf{T}\left(1, \mathbf{p}_{1}, \mathbf{p}_{2}\right)=[0,0,1]^{T}$, which is equivalent to final orientation angles yaw $\psi\left(1, \mathbf{p}_{1}, \mathbf{p}_{2}\right)=\pi / 2$ rad and pitch $\theta\left(1, \mathbf{p}_{2}\right)=-\pi / 2 \mathrm{rad}$, as stated in Eq. (16). Notice that the projection of a Cb3D curve onto $X Y$ plane (horizontal plane $Z=0)$ is the planar clothoid $\mathbf{C}_{1}\left(s, \mathbf{p}_{1}, \mathbf{p}_{2}\right)$, as can be seen in Fig. $2 b$. 


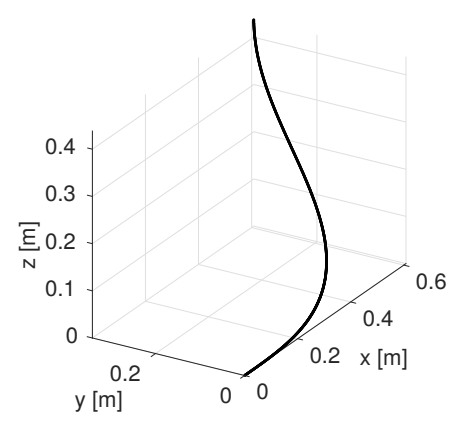

(a) $\mathbf{C}\left(s, \mathbf{p}_{1}, \mathbf{p}_{2}\right)$

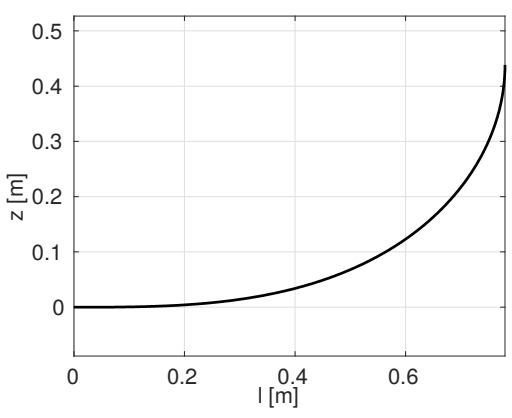

(c) $\mathbf{C}_{2}\left(s, \mathbf{p}_{2}\right)$

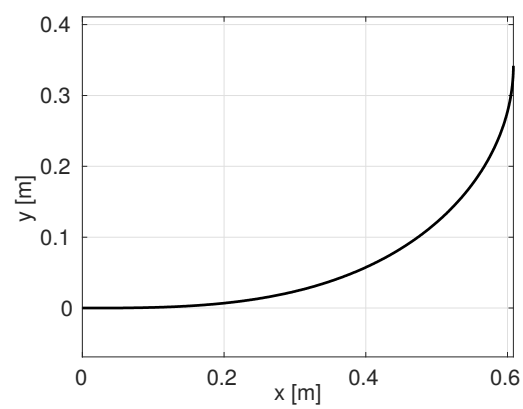

(b) Projection onto $X Y$ plane

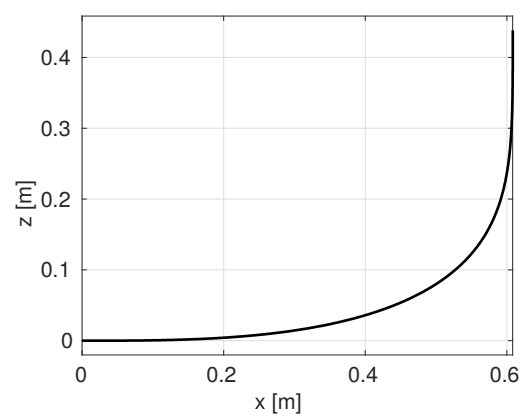

(d) Projection onto $X Z$ plane

Fig. 2 Cb3D curve $\mathbf{C}\left(s, \mathbf{p}_{1}, \mathbf{p}_{2}\right)$, for $s \in[0,1] \mathrm{m}, \mathbf{p}_{2}=\{0,0,-\pi\}$ and $\mathbf{p}_{1}=\left\{0,0, \pi / C^{2}\left(1, \mathbf{p}_{2}\right)\right\}$, with $l \equiv C\left(s, \mathbf{p}_{2}\right)$.

On the other hand, $\mathbf{C}_{2}\left(s, \mathbf{p}_{2}\right)$ is a second clothoid produced by unrolling the Cb3D curve onto a virtual vertical plane defined by the length of the horizontal clothoid $l \equiv C\left(s, \mathbf{p}_{2}\right)$ as base and $z \equiv \mathcal{S}\left(s, \mathbf{p}_{2}\right)$ as height, shown in Fig. 2c. It is interesting to remark that the projection of a $\mathrm{Cb} 3 \mathrm{D}$ curve onto $X Z$ plane is not strictly a planar clothoid, but a 2D clothoid flattened along its $x$ component, as can be observed in Fig. 2d. Based on the symmetry property (C) of Lemma III.1. a Cb3D curve can be defined for $s \in \mathbb{R}$ in any of the 8 octants in $\mathbb{R}^{3}$ space, as depicted in Fig. 3, where a Cb3D curve parametrized as in Fig. 2 has been used.

\section{Synthesis}

As explained in the problem statement, the parameter vector $\mathbf{p}$ is composed by two sets of parameters: $\mathbf{p}_{0}$ related to the initial conditions of the curve and $\tilde{\mathbf{p}}$ the actual parameters to design. In particular, the Cb3D curve uses $\mathbf{p}_{0}:=\left\{\psi_{0}, \theta_{0}, \epsilon_{0}, v_{0}\right\}$ and $\tilde{\mathbf{p}}:=\{\mu, \rho\}$. So, let's recall that our aim is to find $\tilde{\mathbf{p}}^{*}:=\left\{\mu^{*}, \rho^{*}\right\}$ such that Eq. (11) is minimized. Thus, by abusing of notation we denote $\mathbf{C}\left(s, \mathbf{p}^{*}\right) \equiv \mathbf{C}\left(s, \mathbf{p}_{0}, \tilde{\mathbf{p}}^{*}\right) \equiv \mathbf{C}\left(s, \mathbf{p}_{1}^{*}, \mathbf{p}_{2}^{*}\right)$, with $\mathbf{p}_{1}^{*}:=\left\{\psi_{0}, \epsilon_{0}, \mu^{*}\right\}$ and $\mathbf{p}_{2}^{*}:=\left\{\theta_{0}, v_{0}, \rho^{*}\right\}$.

Lemma III.2 Given an arbitrary target tangent vector $\mathbf{T}^{*}$, defined by final angles $\theta^{*} \in[-\pi / 2, \pi / 2] \mathrm{rad}$ and $\psi^{*} \in[-\pi, \pi]$ $\mathrm{rad}$, and given values of arc-length $s>0$, and parameters $\mathbf{p}_{0}=\left\{\psi_{0}, \theta_{0}, \epsilon_{0}, v_{0}\right\}$ which can be obtained from initial orientation, curvature, and torsion, there is only one pair of parameters $\tilde{\mathbf{p}}^{*}=\left\{\mu^{*}, \rho^{*}\right\}$ that synthesize a Cb3D curve $\mathbf{C}\left(s, \mathbf{p}_{1}^{*}, \mathbf{p}_{2}^{*}\right)$ reaching $\mathbf{T}\left(s, \mathbf{p}_{1}^{*}, \mathbf{p}_{2}^{*}\right)=\mathbf{T}^{*}$. 


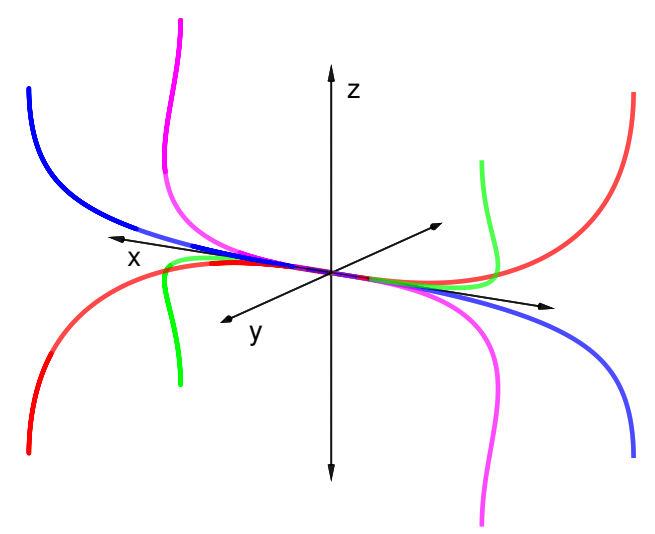

Fig. 3 Family of symmetric unitary Cb3D curves covering all 8 octants, parametrized as in Fig. 2

Proof For a given value of arc-length $s>0$, and final angles $\left\{\theta^{*}, \psi^{*}\right\}$, using Eqs. (13) and (14], the parameters in $\tilde{\mathbf{p}}^{*}$ can be computed as

$$
\begin{aligned}
\rho^{*} & =\frac{2\left(\theta^{*}-\theta_{0}-v_{0} s\right)}{s^{2}} \\
\mu^{*} & =\frac{2\left(\psi^{*}-\psi_{0}-\epsilon_{0} C\left(s, \mathbf{p}_{2}^{*}\right)\right)}{C^{2}\left(s, \mathbf{p}_{2}^{*}\right)}
\end{aligned}
$$

Therefore, the solution is unique and synthesizes a Cb3D curve expressed as in Eqs. 41] and 42) of Appendix A

$$
\mathbf{C}\left(s, \mathbf{p}_{1}^{*}, \mathbf{p}_{2}^{*}\right):=\left\{\begin{array}{c}
\int_{0}^{C\left(s, \mathbf{p}_{2}^{*}\right)} \cos \left(\psi_{0}+\epsilon_{0} \xi+\frac{\mu^{*}}{2} \xi^{2}\right) d \xi \\
\int_{0}^{C\left(s, \mathbf{p}_{2}^{*}\right)} \sin \left(\psi_{0}+\epsilon_{0} \xi+\frac{\mu^{*}}{2} \xi^{2}\right) d \xi \\
-\int_{0}^{s} \sin \left(\theta_{0}+v_{0} \xi+\frac{\rho^{*}}{2} \xi^{2}\right) d \xi
\end{array}\right.
$$

with

$$
\mathcal{C}\left(s, \mathbf{p}_{2}^{*}\right):=\int_{0}^{s} \cos \left(\theta_{0}+v_{0} \xi+\frac{\rho^{*}}{2} \xi^{2}\right) d \xi
$$

Lemma III.3 Given an arbitrary final tangent vector $\mathbf{T}^{*}$, and any pair of arc-lengths $s_{1}>0$ and $s_{2}>0$, the solutions to reach $\mathbf{T}^{*}$, computed as in LemmaIII.2 (Eqs. (19) and (20)), are $\tilde{\mathbf{p}}_{1}^{*}:=\left\{\mu_{1}^{*}, \rho_{1}^{*}\right\}$ and $\tilde{\mathbf{p}}_{2}^{*}:=\left\{\mu_{2}^{*}, \rho_{2}^{*}\right\}$ for each arc-length. Then, the ratio $\eta^{*}:=\rho_{1}^{*} / \mu_{1}^{*}=\rho_{2}^{*} / \mu_{2}^{*}$ is a constant value for $\forall s_{1} \in \mathbb{R}^{+}$and $\forall s_{2} \in \mathbb{R}^{+}$iff $v_{0}=0$ and $\epsilon_{0}=0$.

Proof From property (A) of Lemma 3.1 (Eqs. (32) and (33) in Appendix A), it is straightforward to see that scaling arc-length by a factor $\lambda$, so that $s_{2}=\lambda s_{1}$, requires a new pair of parameters $\left\{\rho_{2}^{*}, \mu_{2}^{*}\right\}=\left\{\rho_{1}^{*} / \lambda^{2}, \mu_{1}^{*} / \lambda^{2}\right\}$, whose ratio $\eta^{*}$ 


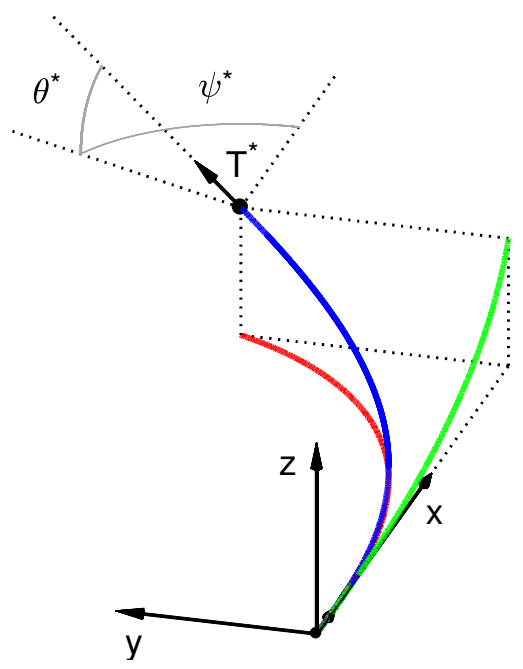

(a)

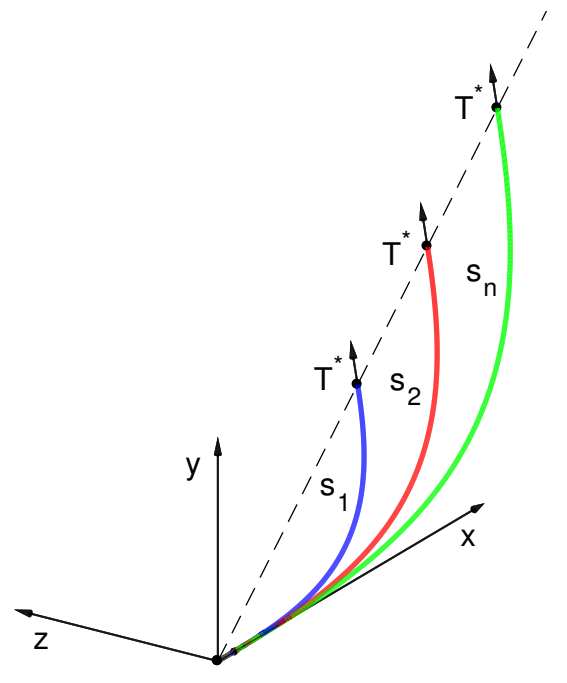

(b)

Fig. 4 Examples of Cb3D curves reaching arbitrary final tangent vector $\mathbf{T}^{*}$ : (a) Cb3D curve (blue) and its projections onto $X Y$ plane (red) and $X Z$ plane (green). (b) Family of Cb3D curves with different arc-lengths.

remains constant.

$$
\begin{aligned}
& \rho_{2}^{*}=\frac{2\left(\theta^{*}-\theta_{0}\right)}{s_{2}^{2}}=\frac{2\left(\theta^{*}-\theta_{0}\right)}{\lambda^{2} s_{1}^{2}}=\frac{\rho_{1}^{*}}{\lambda^{2}} \\
& \mu_{2}^{*}=\frac{2\left(\psi^{*}-\psi_{0}\right)}{C^{2}\left(s_{2}, \mathbf{p}_{2}^{*}\right)}=\frac{2\left(\psi^{*}-\psi_{0}\right)}{C^{2}\left(\lambda s_{1}, \lambda^{-1} \cdot \mathbf{p}_{1}^{*}\right)}=\frac{2\left(\psi^{*}-\psi_{0}\right)}{\lambda^{2} C^{2}\left(s_{1}, \mathbf{p}_{1}^{*}\right)}=\frac{\mu_{1}^{*}}{\lambda^{2}}
\end{aligned}
$$

Figure $4 a$ shows a Cb3D curve reaching an arbitrary final tangent vector $\mathbf{T}^{*}$. It can be observed that final angles $\theta^{*}$ and $\psi^{*}$ are the deflection angles of the tangent vector with respect to planes $X Y$ and $X Z$, as stated in the orientation property (D) of Lemma III.1 On the other hand, Fig. 4b illustrates Lemma III.3, closely related to the scaling property (A) of Lemma III.1, where multiplying a Cb3D curve by a scale factor does not modify its shape and final orientation.

Lemma III.4 Given an arbitrary target position vector $\mathbf{C}^{*}:=\left\{x^{*}, y^{*}, z^{*}\right\}$, with arc-length $s>0$ and initial parameters $\mathbf{p}_{0}=\{0,0,0,0\}$, there is only one pair of parameters $\tilde{\mathbf{p}}^{*}=\left\{\mu^{*}, \rho^{*}\right\}$ that synthesize a Cb3D curve as a solution to the following position minimization problem

$$
\tilde{\mathbf{p}}^{*}=\arg _{\tilde{\mathbf{p}}} \min \left\|\mathbf{C}^{*}-\mathbf{C}\left(s, \mathbf{p}_{0}, \tilde{\mathbf{p}}\right)\right\|
$$


where, for $\mathbf{C}^{*}$ to be reachable by the $\mathrm{Cb} 3 \mathrm{D}$ curve, the following conditions are required

$$
\begin{aligned}
& \left|y^{*}\right| \leq 1.765042236 \cdot x^{*} \\
& \left|z^{*}\right| \leq 0.561947501 \cdot l^{*}
\end{aligned}
$$

being $l^{*}$ the arc-length of a planar clothoid $\mathbf{C}_{1}\left(l^{*}, \mathbf{p}_{1}\right)=\left\{x^{*}, y^{*}\right\}$, so that the condition that constrains component $z^{*}$ depends on the solution of the projection of $\mathbf{C}^{*}$ onto $X Y$ plane.

Proof If a solution for $\mathbf{C}^{*}=\mathbf{C}\left(s, \mathbf{p}_{0}, \tilde{\mathbf{p}}^{*}\right)$ can be found, therefore such solution will minimize 25]. So, let's define a unitary planar clothoid $\mathbf{C}_{1}\left(s_{1}, \mathbf{p}_{1}\right)$ as in Eq. 9], with $\mathbf{p}_{1}=\{0,0, \pi\}$. The goal is to solve the following problem

$$
\frac{\left|y^{*}\right|}{x^{*}}=\frac{\mathcal{S}\left(s_{1}, \mathbf{p}_{1}\right)}{C\left(s_{1}, \mathbf{p}_{1}\right)}
$$

being $s_{1}$ the arc-length of $\mathbf{C}_{1}\left(s_{1}, \mathbf{p}_{1}\right)$ contained in the horizontal plane, where the solution $s_{1}^{*}$ can be found using any root finding algorithm, and it is unique iff condition $(26)$ is satisfied. Such condition establishes the maximum ratio between coordinates $X$ and $Y$, which is produced when the line tangent to the planar clothoid passes through the origin [51], where for a unitary clothoid it happens at $s_{1}=1.634577 \mathrm{~m}$, so that $\mathbf{C}_{1}(1.634577,\{0,0, \pi\}) \approx\{0.345860,0.610458\} \mathrm{m}$.

From property (A) of Lemma 3.1 (Eqs. (32) and (33) in Appendix A), the horizontal unitary clothoid contained in plane $X Y$, found as a solution to Eq. [28], can be scaled to the original target position coordinates, so that $\left\{x^{*},\left|y^{*}\right|\right\}=K_{1} \mathbf{C}\left(s_{1}^{*}, \mathbf{p}_{1}\right)$, with scaled arc-length $l^{*}=K_{1} s_{1}^{*}$, being $K_{1}$ the homothety factor of the first planar clothoid, which can be easily computed as

$$
K_{1}=\frac{x^{*}}{C\left(s_{1}^{*}, \mathbf{p}_{1}\right)}=\frac{\left|y^{*}\right|}{\mathcal{S}\left(s_{1}^{*}, \mathbf{p}_{1}\right)}
$$

Now, let's repeat the previous steps for a second unitary planar clothoid $\mathbf{C}_{2}\left(s_{2}, \mathbf{p}_{2}\right)$ in the vertical plane, with $\mathbf{p}_{2}=\{0,0, \pi\}$. We aim to find a solution to the following equality

$$
\frac{\left|z^{*}\right|}{l^{*}}=\frac{\mathcal{S}\left(s_{2}, \mathbf{p}_{2}\right)}{C\left(s_{2}, \mathbf{p}_{2}\right)}
$$

being $s_{2}$ the arc-length of $\mathbf{C}_{2}\left(s_{2}, \mathbf{p}_{2}\right)$, where the solution $s_{2}^{*}$ can be found by any root finding algorithm, being unique iff condition (27) is satisfied, where for a unitary clothoid it happens at $s_{2}=1 \mathrm{~m}$, so that $\mathbf{C}_{2}(1,\{0,0, \pi\}) \approx$ $\{0.779893,0.438259\} \mathrm{m}$. Again, using property (A) of Lemma 3.1, the unitary clothoid can be scaled $\left\{l^{*},\left|z^{*}\right|\right\}=$ 
$K_{2} \mathbf{C}\left(s_{2}^{*}, \mathbf{p}_{2}\right)$, where $s^{*}=K_{2} s_{2}^{*}$ is the arc-length of the vertical planar clothoid and $K_{2}$ its homothety factor

$$
K_{2}=\frac{l^{*}}{C\left(s_{2}^{*}, \mathbf{p}_{2}\right)}=\frac{\left|z^{*}\right|}{\mathcal{S}\left(s_{2}^{*}, \mathbf{p}_{2}\right)}
$$

Using planar clothoids properties, $\tilde{\mathbf{p}}^{*}=\left\{\mu^{*}, \rho^{*}\right\}$ can be computed as $\mu^{*}=\operatorname{sgn}\left(y^{*}\right) \frac{\pi}{K_{1}^{2}}$ and $\rho^{*}=-\operatorname{sgn}\left(z^{*}\right) \frac{\pi}{K_{2}^{2}}$, where $\operatorname{sgn}(\bullet)$ is the signum function.

As a conclusion, Lemmas III.2, III.3 and III.4 imply that a Cb3D curve can reach a target position $\mathbf{C}^{*}$ in the space with one and only one orientation $\mathbf{T}^{*}$. On the contrary, a target tangent vector $\mathbf{T}^{*}$ can be reached by a Cb3D curve in multiple positions, depending on the arc-length $s>0$. However, the final position of such solutions lies in a straight line, since any solution to the problem has the same shape and is related by a scaling factor $\lambda$.

Finally, it is obvious that the solution to the orientation minimization problem to reach target tangent vector $\mathbf{T}^{*}$, which is stated in Eq. (11) and given by the pair of design parameters $\tilde{\mathbf{p}}^{*}=\left\{\mu^{*}, \rho^{*}\right\}$, is the same as the solution obtained when solving the position minimization problem in Eq. 25], iff arc-length $s=s^{*}$, where $s^{*}$ is the length of the Cb3D curve designed to reach target position $\mathbf{C}^{*}$. Therefore, in path planning problems, due to geometric constraints only final arbitrary orientation or position can be reached by a Cb3D curve, but not both simultaneously.

\section{Comparison: C3D vs. Cb3D}

In this section we will compare the proposed clothoid-based 3D curve (Cb3D) and the pure 3D clothoid (C3D) introduced in [37]. Both curves are considered geometric curves and therefore can be directly compared considering not just only their geometric properties, but also their kinematic properties as well as their computational time requirements. An example of singular case for $\mathrm{C} 3 \mathrm{D}$ is also shown and explained in order to compare both curves.

The authors of [37] used a fixed step Euler integration method [49]. In this sense, to speed up computation, such method has been improved by implementing an algorithm to solve ordinary differential equations with variable step [49]. Besides, values of curvature sharpness $\sigma_{\kappa}$ and torsion sharpness $\sigma_{\tau}$ required to join initial and final orientations cannot be known a priori, so an optimization procedure using Gradient-descent algorithm is required [37]. Regarding the parameter initialization of the optimizer, initial sharpness pair $\left(\sigma_{\kappa_{0}}\right.$ and $\left.\sigma_{\tau_{0}}\right)$ is set to the solution proposed in our method. Although they do not represent the same variables, we could verify that this initialization is better than random or naive initializations, in terms of computation time and final accuracy. On the other hand, initial values of curvature and torsion are set to $\kappa_{0}=0$ and $\tau_{0}=0$, and they are not modified during optimization procedure, which differs from [37]. This modification is due to the fact that we want to solve the problem of a fixed-wing airplane following a straight line, which in a certain time instant needs to perform a maneuver to turn and change its height and direction of movement. Therefore, $\kappa_{0}=\tau_{0}=0 \mathrm{rad} / \mathrm{m}$ is required as a boundary condition for the problem to be solved.

It is interesting to remark that, other approaches, based on parametric curves, such as Bézier, Splines or B-spline 
curves were considered as well, however as stated by [18], we found they were very sensitive to control points initialization and in order to be comparable with clothoid-based approaches they were required to minimize the jerk and to impose specific constraints on the curves. The results were not satisfactory and are not shown for brevity.

The test performed consists of planning a curve joining the initial tangent vector $\mathbf{T}(0)=\left[\begin{array}{lll}1 & 0 & 0\end{array}\right]^{T}$, equivalent to $\mathbf{p}_{0}=\{0,0,0,0\}$, to a final arbitrary $\mathbf{T}^{*}$, whose pitch and yaw angles are randomly drawn from a uniform distribution $\theta^{*} \sim \mathcal{U}(0, \pi / 2) \mathrm{rad}$ and $\psi^{*} \sim \mathcal{U}(0, \pi / 2)$ rad. A total of $n=10^{3}$ random combinations of samples have been generated. For all cases the curve length is set to $s=1 \mathrm{~m}$.

\section{Computation Time and Accuracy}

As shown in Table 1, computation time of a Cb3D curve is much lower (around $10^{-4}$ times smaller) than for the case of C3D. The main reason is that Cb3D curves can be computed in closed-form based on C2D approximations [45, 46], while C3D computation requires numerical integration of Eqs. (1) and (2) [37], which is much slower.

Now, analyzing the worst case of computation time, for C3D it is around $0.4 \mathrm{~s}$, while the maximum computation time of a Cb3D curve is approximately $700 \mu$ s. Therefore, our method can be used to compute 3D smooth curves in real-time. Note that all processing has been done in a computer with Intel Core i7-6700HQ 2.60GHz processor and 16GiB DDR4 memory, using an implementation of both methods in MATLAB ${ }^{\circledR}$ R2017a.

Table 1 Computation time and accuracy: Cb3D vs. C3D.

\begin{tabular}{lcccc}
\hline \hline Performance Metric & Space curve & Mean & Standard deviation & Worst case \\
\hline \multirow{2}{*}{ Computation time [ms] } & C3D & 282.241 & 27.670 & 413.251 \\
\cline { 2 - 5 } & Cb3D & $32.991 \cdot 10^{-3}$ & $28.499 \cdot 10^{-3}$ & 0.731 \\
\hline \multirow{2}{*}{ Accuracy [m] } & C3D & $7.595 \cdot 10^{-15}$ & $49.053 \cdot 10^{-15}$ & $8.982 \cdot 10^{-13}$ \\
\cline { 2 - 5 } & Cb3D & $1.764 \cdot 10^{-16}$ & $1.399 \cdot 10^{-16}$ & $8.006 \cdot 10^{-16}$ \\
\hline \hline
\end{tabular}

Regarding the accuracy, a Cb3D curve has the same order of magnitude as planar clothoids [45], whilst the accuracy of $\mathrm{C} 3 \mathrm{D}$ depends on the stop conditions of the optimization method. Thus, the more accurate, the higher the computation time, and vice versa. In order to obtain the results of Table 1 , the stop condition was intentionally set to $e=10^{-15} \mathrm{~m}$ to do a fair comparison, where $e=\sqrt{\left(\theta^{*}-\theta\right)^{2}+\left(\varphi^{*}-\varphi\right)^{2}}$ is the error between the target orientation $\left\{\theta^{*}, \varphi^{*}\right\}$ and the final orientation $\{\theta, \varphi\}$ reached by the generated C3D.

\section{Geometry and Kinematics}

Some geometric and kinematic metrics are evaluated in this section. On the one hand, geometric metrics, which only 


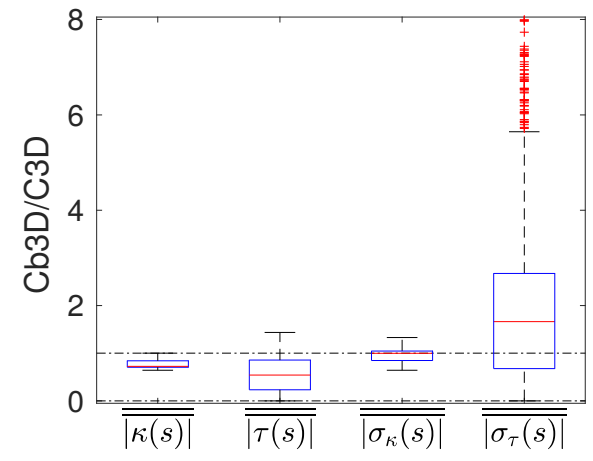

(a) Maximum of geometric variables

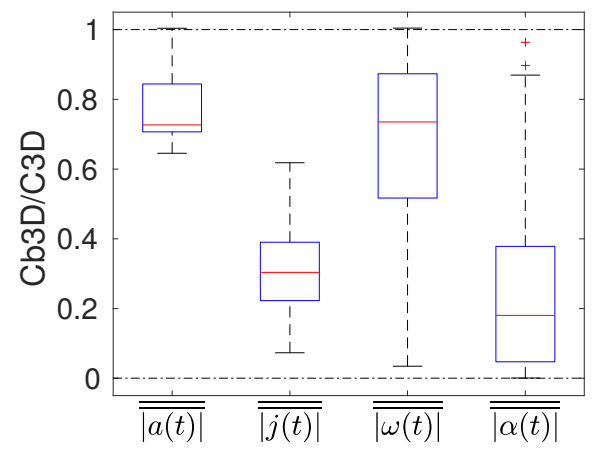

(c) Maximum of kinematic variables

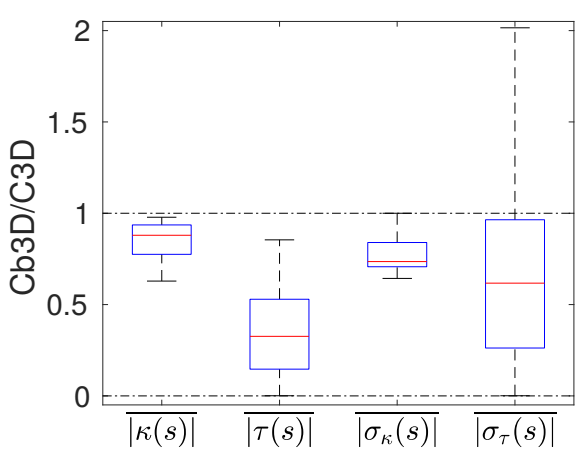

(b) Mean of geometric variables

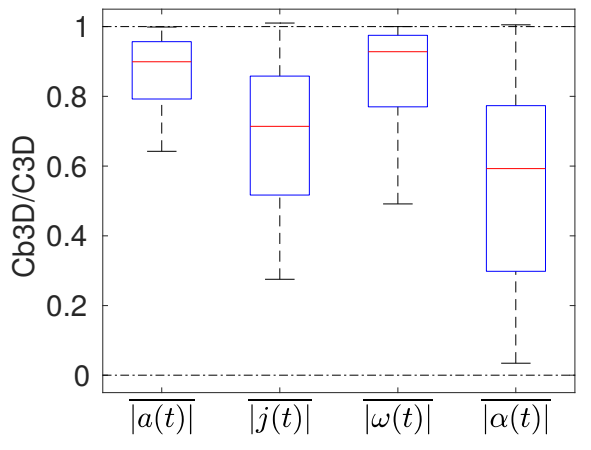

(d) Mean of kinematic variables

Fig. 5 Ratio Cb3D/C3D of metrics related to geometry (a,b) and kinematics (c,d), with maximum absolute values (a,c) and mean absolute values $(\mathrm{b}, \mathrm{d})$.

depend on arc-length $s$, comprise mean and maximum values of curvature $\kappa(s)$, torsion $\tau(s)$, and their derivatives, curvature sharpness $\sigma_{\kappa}(s)$ and torsion sharpness $\sigma_{\tau}(s)$. On the other hand, kinematic variables, which depend on time $t$, are mean and maximum values of acceleration $a(t)$, jerk $j(t)$, angular velocity $\omega(t)$, and angular acceleration $\alpha(t)$. Kinematic variables have been computed assuming a constant linear velocity $v e l=1 \mathrm{~m} / \mathrm{s}$, so that curve length is time-dependent $s(t)=t$. Besides, since all four kinematic variables are vectors, metrics have been computed as the 2-norm of such vectors. Note that $\overline{\overline{|\bullet|}}$ means maximum values, while $\overline{|\bullet|}$ means average values.

For each random case, metrics have been computed as a ratio between absolute values of the variables obtained when computing $\mathrm{Cb} 3 \mathrm{D}$ with respect to $\mathrm{C} 3 \mathrm{D}$, so that $r_{i}:=\left|m_{i_{C b 3 D}}\right| /\left|m_{i_{C 3 D}}\right|$ is the ratio of the $i$-th metric, where $m_{i_{C b 3 D}}$ is the metric obtained with $\mathrm{Cb} 3 \mathrm{D}$ and $m_{i_{C 3 D}}$ is the metric obtained with $\mathrm{C} 3 \mathrm{D}$.

From Fig. 5a, it can be concluded that $\mathrm{Cb3D}$ tends to have lower maximum values of curvature $\overline{\overline{|\kappa|}}(100 \%$ of cases) and torsion $\overline{\overline{|\tau|}}(86.2 \%$ of cases $)$. Moreover, the maximum value of curvature sharpness $\overline{\overline{\left|\sigma_{\kappa}\right|}}$ is very similar according to the box-whiskers diagram, although slightly better for $\mathrm{Cb} 3 \mathrm{D}$ computation (50.8\% of cases). However, the maximum value of torsion sharpness $\overline{\overline{\left|\sigma_{\tau}\right|}}$ is worse for $\mathrm{Cb} 3 \mathrm{D}$ (only in $34.3 \%$ of cases $\mathrm{Cb} 3 \mathrm{D}$ is better). This phenomenon is explained later in a graphical example of a particular case representing this situation.

In Fig. $5 \mathrm{~b}$, the average values of geometric variables show that $\mathrm{Cb} 3 \mathrm{D}$ tends to have lower mean values in all four 


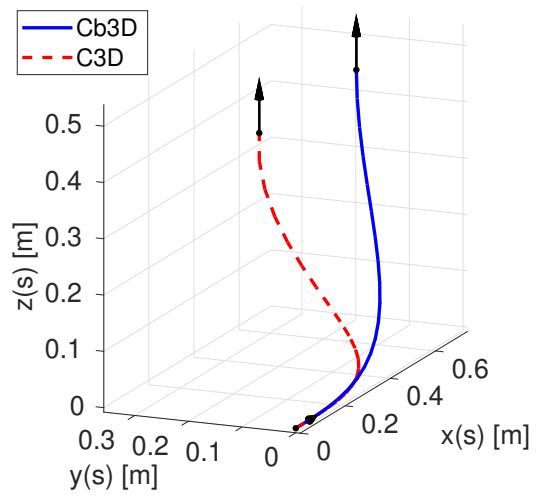

(a) Trajectory
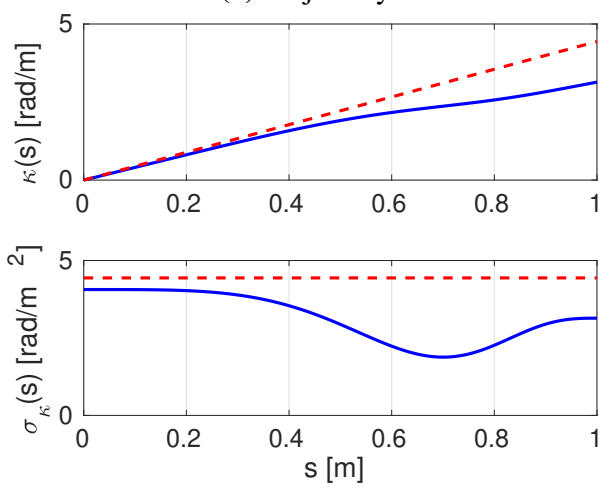

(c) Curvature and its sharpness
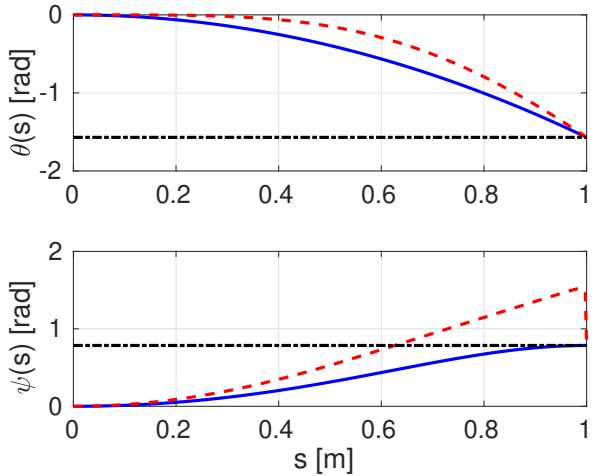

(b) Euler angles
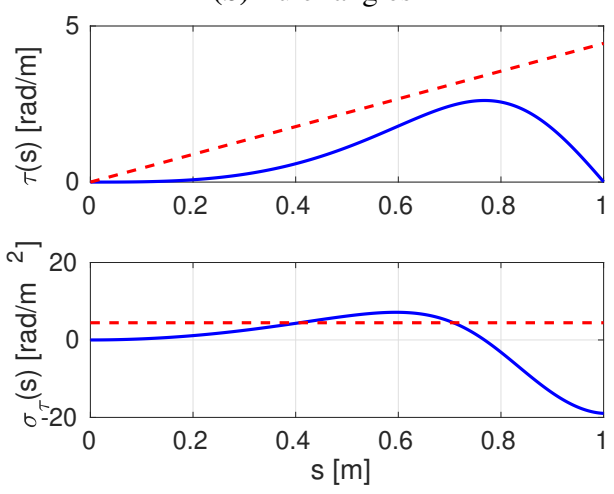

(d) Torsion and its sharpness

Fig. 6 Singular case to reach final orientation $\theta^{*}=-\pi / 2 \mathrm{rad}$ and $\psi^{*}=\pi / 4 \mathrm{rad}$ : Cb3D (blue) vs. C3D (dashed red).

metrics: $\overline{|\kappa|}\left(100 \%\right.$ of cases), torsion $\overline{|\tau|}$ (100\% of cases), curvature sharpness $\overline{\left|\sigma_{K}\right|}$ (100\% of cases), and torsion sharpness $\overline{\left|\sigma_{\tau}\right|}$ (77.1\% of cases). This happens because, as explained later through a graphical example, the Cb3D has lower values most of the time. However, in some particular cases the maximum value of torsion sharpness increases very much, which has 3 different effects: 1$)$ maximum torsion sharpness is much higher in $\mathrm{Cb} 3 \mathrm{D}$ than $\mathrm{C} 3 \mathrm{D}\left(r_{i}>1\right.$ for $\left.\overline{\overline{\left|\sigma_{\tau}\right|}}\right)$, 2) which might imply that the average torsion sharpness of Cb3D surpass the value of $\mathrm{C} 3 \mathrm{D}\left(r_{i}>1\right.$ for $\left.\overline{\left|\sigma_{\tau}\right|}\right)$, and 3) the maximum torsion of Cb3D might also increase to be higher than $\mathrm{C} 3 \mathrm{D}\left(r_{i}>1\right.$ for $\left.\overline{\overline{|\tau|}}\right)$.

Regarding kinematic variables, Fig. 5c shows that maximum values tend to be lower in Cb3D curve, since most ratios are $r_{i}<1$ : acceleration $\overline{\overline{|a|}}$ ( $96.6 \%$ of cases), jerk $\overline{\overline{|j|}}$ (100\% of cases), angular velocity $\overline{\overline{|\omega|}}$ ( $96.5 \%$ of cases), and angular acceleration $\overline{\overline{|\alpha|}}$ (100\% of cases). Something similar happens for ratios of mean values: acceleration $\overline{|a|}$ (100\% of cases), jerk $\overline{|j|}$ (95.6\% of cases), angular velocity $\overline{|\omega|}$ (100\% of cases), and angular acceleration $\overline{|\alpha|}$ (97.3\% of cases).

\section{Singular case}

Figure 6 shows a singular case in which both curves converge to final orientation $\theta^{*}=-\pi / 2$ and $\psi^{*}=\pi / 4$ (gimbal effect of Euler angles with singularity $\theta^{*} \rightarrow \pm \pi / 2 \mathrm{rad}$ ), as depicted in Fig. $6 \mathrm{~b}$. In this particular case, it can be observed 


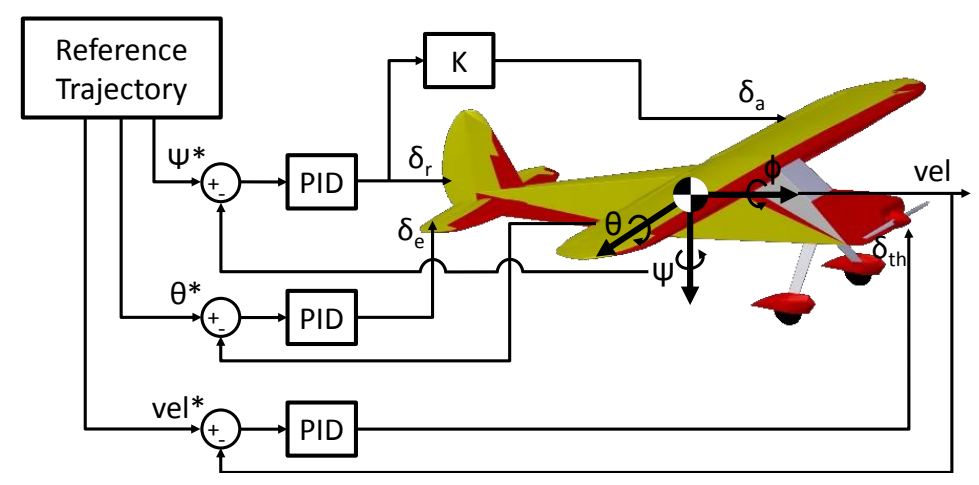

Fig. 7 Model of Kadett 2400, with variables definition and control scheme.

that C3D (dashed red line) needs to correct the yaw angle very abruptly, while Cb3D solves the same problem with a smoother solution based on properties (j) and (k) of LemmaIII.1.

Regarding curvature and torsion, Figs. 6c and 6d show that for a Cb3D curve these variables are non-linear, since their sharpness are not constant values. This result implies that the proposed clothoid-based three-dimensional curve (Cb3D) is not claimed to be a pure three-dimensional clothoid (C3D), but a smooth space curve based on a composition of two-dimensional clothoids (C2D).

\section{Simulation results}

Our curve proposal has been validated in a UAV attitude planning application using the simulator FlightGear 2018, with the dynamic model of the fixed-wing airplane Kadett 2400 [52], depicted in Fig. 7] The system has 4 inputs to control the aerodynamic surfaces and speed: $\delta_{e}$ (elevator), $\delta_{a}$ (aileron), $\delta_{r}$ (rudder) and $\delta_{t h}$ (throttle). Fig. 7 also shows the control structure used to drive the aircraft. Given some initial conditions (current tangent vector T) and a target configuration (final tangent vector $\mathbf{T}^{*}$ ), a reference trajectory is generated in a local planning framework for heading-attitude control architectures, which can be used for obstacle avoidance.

The goal is to keep a constant velocity $v e l^{*}$ along the trajectory, which is accomplished by a PID controller that affects the throttle. Besides, the profiles $\theta^{*}$ and $\psi^{*}$ of the generated trajectory are used as a reference input for two more PID controllers. The first PID controls the elevator, which affects the pitch orientation. Whilst the output of the second PID is applied to the rudder, which affects the yaw orientation and is used to compensate little errors when tracking the reference yaw $\psi^{*}$. Besides, the output of such PID controller is multiplied by a gain $K$ to control the aileron, which directly affects the roll orientation, but also makes the aircraft turn in yaw.

Some results of the experiments carried out in FlightGear simulator are shown in Figs. 8 and 9 . The reference velocity is set to $v e l^{*}=18 \mathrm{~m} / \mathrm{s}$, with a sampling period of $T_{s}=20 \mathrm{~ms}$ to control the airplane. Since the time to perform the maneuver is set to $t=10 \mathrm{~s}$, the length of the reference trajectory is $s=180 \mathrm{~m}$. For this particular case, the target tangent vector $\mathbf{T}^{*}$ is defined by final angles pitch $\theta^{*}=-0.6 \mathrm{rad}$ and yaw $\psi^{*}=0.2 \mathrm{rad}$. 


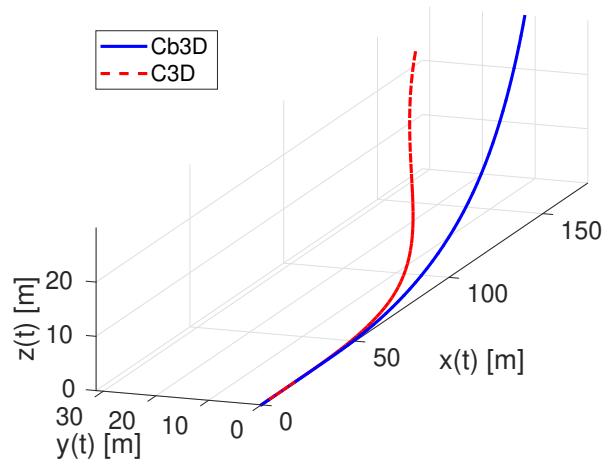

(a) Trajectory
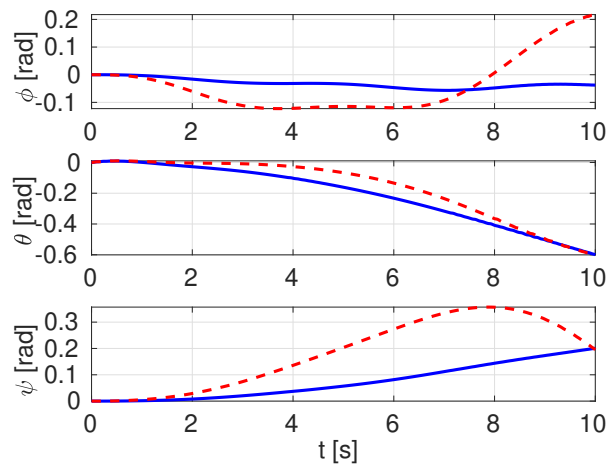

(b) Pitch and yaw angles

Fig. 8 Trajectories followed by the UAV with different references to reach final orientation $\theta^{*}=-0.6 \mathrm{rad}$ and $\psi^{*}=0.2 \mathrm{rad}$ : $\mathrm{Cb} 3 \mathrm{D}$ (blue) vs. C3D (dashed red).

Fig. 8a shows that the aircraft follows a completely different trajectory depending on the curve used as reference. In Fig. $8 \mathrm{~b}$ it can be seen that, in both cases, the UAV starts at $t=0 \mathrm{~s}$ with initial roll $\varphi(0)=0$, pitch $\theta(0)=0$ and yaw $\psi(0)=0$ (relative to its current local frame, used as reference coordinate system for the planning task). The Cb3D reference (blue line) is much smoother and more intuitive, since it avoids unnecessary turnings. Actually, the main drawback of using a C3D as reference (dashed red line) is the fact that in order to follow such a curve the airplane has to overshoot and turn excessively in yaw, and then compensate by counter turning to reach the target orientation, using roll to stabilize itself. This problem is intrinsic of pure 3D clothoid, since it is a spiral with some particular properties and limitations. On the contrary, all these problems are avoided by the way a clothoid-based 3D curve is generated, because for any target configuration both orientation and position increase monotonically and smoothly, which helps fixed-wing planes track such a reference.

Fig. 9 depicts a sequence of screenshots of the previous simulation at different time instants $t=\{0,3,7,10\} \mathrm{s}$. It can be seen that the UAV starts at $t=0 \mathrm{~s}$ completely horizontal, moving straight in both cases (see Figs. 9a and 9e). Then, it starts to turn to the left by rotating in roll (see Figs. 9b and 9f), while progressively increasing its height (see Figs. 9c and 9g). The last pair of images (see Figs. 9d and 9h) show the UAV in the last configuration at $t=10 \mathrm{~s}$, with pitch $\theta(10)=-0.6 \mathrm{rad}$ and yaw $\psi(10)=0.2 \mathrm{rad}$. Even though both trajectories guide the vehicle to the same target tangent vector, notice that the $\mathrm{C} 3 \mathrm{D}$ planning forces the vehicle to turn much more in roll and yaw, than for the case of $\mathrm{Cb} 3 \mathrm{D}$ planning. This unnecessary turning might difficult the control task and produce some undesired oscillatory or even unstable behavior in the airplane. For instance, UAV's final attitude for the C3D reference has a positive roll (see Figs. $8 \mathrm{~b}$ and $9 \mathrm{~d}$, which makes the airplane turn to the right, but the target yaw was a positive value so the turning tendency should be to the left. This situation is one of the potential cases where a $\mathrm{Cb} 3 \mathrm{D}$ curve outperforms a pure 3D clothoid in motion planning problems for fixed-wing UAVs.

The previous example and some more target orientations are shown and explained in the following video. The first 


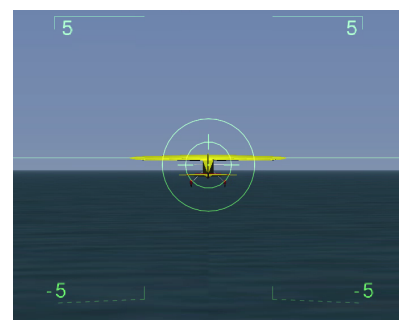

(a) $t=0 \mathrm{~s}(\mathrm{C} 3 \mathrm{D})$

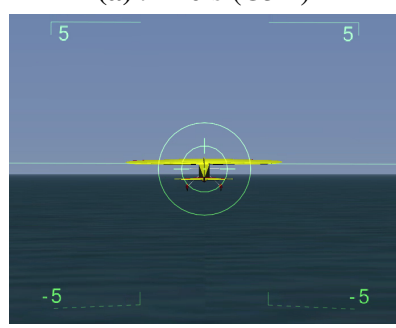

(e) $t=0 \mathrm{~s}(\mathrm{Cb} 3 \mathrm{D})$

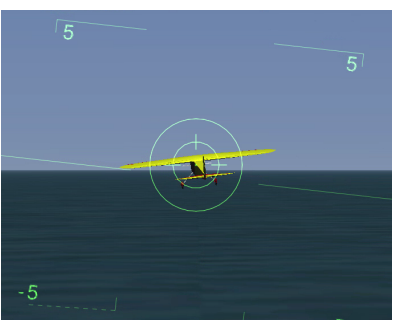

(b) $t=3 \mathrm{~s}(\mathrm{C} 3 \mathrm{D})$

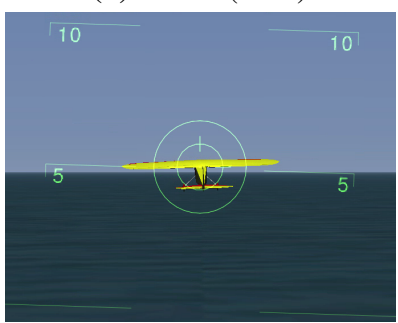

(f) $t=3 \mathrm{~s}(\mathrm{Cb} 3 \mathrm{D})$

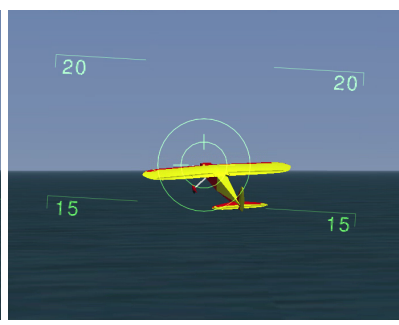

(c) $t=7 \mathrm{~s}(\mathrm{C} 3 \mathrm{D})$

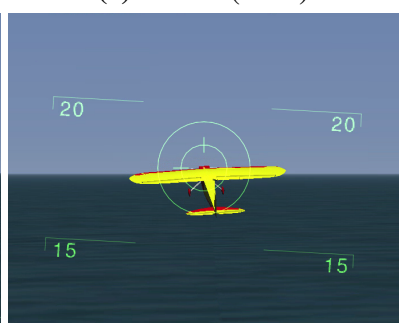

(g) $t=7 \mathrm{~s}(\mathrm{Cb} 3 \mathrm{D})$

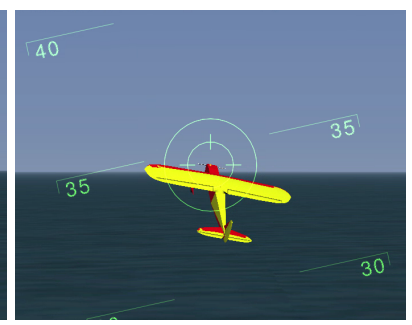

(d) $t=10 \mathrm{~s}(\mathrm{C} 3 \mathrm{D})$

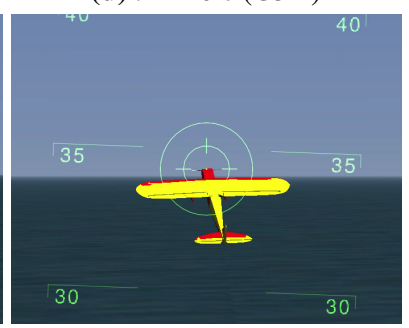

(h) $t=10 \mathrm{~s}(\mathrm{Cb} 3 \mathrm{D})$

Fig. 9 Screenshots of the simulation in FlightGear with final orientation $\theta^{*}=-0.6 \mathrm{rad}$ and $\psi^{*}=0.2 \mathrm{rad}$, using different references: C3D (a-d) and Cb3D curve (e-h).

case is for a final orientation $\theta^{*}=0 \mathrm{rad}$ and $\psi^{*}=\pi \mathrm{rad}$, where both planners generate the same reference, a planar clothoid, so the UAV traces the same trajectory both times. The second example is for target angles $\theta^{*}=-0.2 \mathrm{rad}$ and $\psi^{*}=0.6 \mathrm{rad}$, where the trajectories followed by the airplane are not exactly the same, although they have similar profiles of roll, pitch and yaw. The third case, for $\theta^{*}=-0.6 \mathrm{rad}$ and $\psi^{*}=0.2 \mathrm{rad}$, has been already explained before in detail. Finally, the fourth example is for target orientation $\theta^{*}=-0.9 \mathrm{rad}$ and $\psi^{*}=0 \mathrm{rad}$, and it can be seen that for high values of $\theta^{*}$ with low $\psi^{*}$ the $\mathrm{C} 3 \mathrm{D}$ planner generates poor references, whilst the $\mathrm{Cb} 3 \mathrm{D}$ trajectories generated by the planner are smoother and very intuitive, since they increase monotonically in both position and orientation, which helps the low-level controller track the reference, guiding and stabilizing easily the flying vehicle. So, as a conclusion, the lower the value of $\theta^{*}$ the more similar $\mathrm{C} 3 \mathrm{D}$ and $\mathrm{Cb} 3 \mathrm{D}$ trajectories are. On the contrary, the higher the value of $\theta^{*}$ the more different $\mathrm{C} 3 \mathrm{D}$ and $\mathrm{Cb} 3 \mathrm{D}$ trajectories are, being the $\mathrm{Cb} 3 \mathrm{D}$ curve more intuitive and easy to follow from UAV's navigation perspective.

\section{Conclusions}

Navigation of unmanned aerial vehicles (UAVs) and autonomous underwater vehicles (AUVs) requires generation of reference trajectories that need to be achievable and fast to compute. Space curves can be used to control such vehicles, but most of them are not very intuitive and/or require optimization procedures, which might be not feasible for real-time applications. Specially for the case of an airplane moving forward that needs to turn in order to change its attitude and altitude, for instance to avoid a collision.

A new space curve, coined as clothoid-based three-dimensional curve (Cb3D), has been presented as a composition of two planar clothoids. As proven in the paper, the proposed Cb3D curve can be seen as a generalization of other 
curves such as a straight line, a circular arc, a circular helix or a planar clothoid. They can be scaled and symmetric curves can be easily computed too. More importantly, it has been shown that the Cb3D curve is $C^{\infty}$ smooth, and its position and orientation vectors are monotonic increasing. This property is very helpful for motion-planning, specially in obstacle avoidance problems. In fact, the shape of a Cb3D curve avoids unnecessary turnings, allowing its inclusion as a primitive for more complex path planning problems, where arbitrary initial and final configurations (position and orientation) could be joined smoothly.

Cb3D curves have been compared to pure 3D clothoids (C3D). Our results show that Cb3D curves can be several order of magnitude faster, because they can be approximated in a closed-form with high accuracy and can be computed analytically, which has benefits in terms of computation time for real-time planning. Besides, in the problem of joining two arbitrary orientations in the space, most of geometric and kinematic properties tend to be lower for $\mathrm{Cb} 3 \mathrm{D}$ than for $\mathrm{C} 3 \mathrm{D}$, both in mean and maximum values. Besides, since the solution is unique, a Cb3D curve is not dependent on parameter initialization, contrary to Bézier or B-spline curves, and does not require optimization procedures as 3D clothoids and parametric curves.

Contrary to $\mathrm{C} 3 \mathrm{D}$, in the case of a $\mathrm{Cb} 3 \mathrm{D}$ curve its curvature and torsion evolve non-linearly with respect to curve length. This might look inappropriate from the point of view of differential geometry, but from the perspective of planning and navigation of UAVs and AUVs it is not that relevant, as it has been shown. In addition to this, we used the FlightGear simulator to show some of the benefits of our proposal in motion-planning methods, since the generated trajectory is smooth and has not overshoot on the orientation.

\section{Appendix A: Proof of Lemma III.1}

A Scalability First, we can see that for the planar clothoid, the scalability property holds. For the tangent angle in Eq. (7), it can be seen that $\beta(s, \mathbf{p})=\beta\left(\lambda s, \lambda^{-1} \cdot \mathbf{p}\right)$. Therefore, we can also see that $\mathbf{T}(s, \mathbf{p})=\mathbf{T}\left(\lambda s, \lambda^{-1} \cdot \mathbf{p}\right)$ in Eq. (8) and so $\lambda \mathbf{C}(s, \mathbf{p})=\mathbf{C}\left(\lambda s, \lambda^{-1} \cdot \mathbf{p}\right)$ in Eq. 97 .

Now, we will proof that the same properties hold for Eq. (12). Following the same argumentations, it can clearly seen that $\lambda \mathbf{C}_{1}\left(s, \mathbf{p}_{1}\right)=\mathbf{C}_{1}\left(\lambda s, \lambda^{-1} \cdot \mathbf{p}_{1}\right)$ and $\lambda \mathbf{C}_{2}\left(s, \mathbf{p}_{2}\right)=\mathbf{C}_{2}\left(\lambda s, \lambda^{-1} \cdot \mathbf{p}_{2}\right)$. Therefore, it can also be asserted that

$$
\begin{aligned}
& \lambda C\left(C\left(s, \mathbf{p}_{2}\right), \mathbf{p}_{1}\right)=C\left(\lambda C\left(s, \mathbf{p}_{2}\right), \lambda^{-1} \cdot \mathbf{p}_{1}\right)=C\left(C\left(\lambda s, \lambda^{-1} \cdot \mathbf{p}_{2}\right), \lambda^{-1} \cdot \mathbf{p}_{1}\right) \\
& \lambda \mathcal{S}\left(C\left(s, \mathbf{p}_{2}\right), \mathbf{p}_{1}\right)=\mathcal{S}\left(\lambda C\left(s, \mathbf{p}_{2}\right), \lambda^{-1} \cdot \mathbf{p}_{1}\right)=\mathcal{S}\left(C\left(\lambda s, \lambda^{-1} \cdot \mathbf{p}_{2}\right), \lambda^{-1} \cdot \mathbf{p}_{1}\right) \\
& \quad-\lambda \mathcal{S}\left(s, \mathbf{p}_{2}\right)=-\mathcal{S}\left(\lambda s, \lambda^{-1} \cdot \mathbf{p}_{2}\right)
\end{aligned}
$$

B Symmetry Let's first assert that a planar clothoid is a symmetric curve, such that $\mathbf{C}(s, \mathbf{p})=-\mathbf{C}(-s, \mathbf{q} \cdot \mathbf{p})$, which can be easily proven from Eq. (9) by considering that $\beta(s, \mathbf{p})=\beta(-s, \mathbf{q} \cdot \mathbf{p})$ in Eq. (7) and $\mathbf{T}(s, \mathbf{p})=\mathbf{T}(-s, \mathbf{q} \cdot \mathbf{p})$ in Eq. 
(8) can be asserted too. Therefore, we can also prove that

$$
\begin{aligned}
\mathcal{C}\left(C\left(s, \mathbf{p}_{2}\right), \mathbf{p}_{1}\right) & =-C\left(-C\left(s, \mathbf{p}_{2}\right), \mathbf{q} \cdot \mathbf{p}_{1}\right)=-C\left(C\left(-s, \mathbf{q} \cdot \mathbf{p}_{2}\right), \mathbf{q} \cdot \mathbf{p}_{1}\right) \\
\mathcal{S}\left(C\left(s, \mathbf{p}_{2}\right), \mathbf{p}_{1}\right) & =-\mathcal{S}\left(-C\left(s, \mathbf{p}_{2}\right), \mathbf{q} \cdot \mathbf{p}_{1}\right)=-\mathcal{S}\left(C\left(-s, \mathbf{q} \cdot \mathbf{p}_{2}\right), \mathbf{q} \cdot \mathbf{p}_{1}\right) \\
\mathcal{S}\left(s, \mathbf{p}_{2}\right) & =-\mathcal{S}\left(-s, \mathbf{q} \cdot \mathbf{p}_{2}\right)
\end{aligned}
$$

C Smoothness A $C^{\infty}$ function is differentiable for all degrees of differentiation, i.e. its position vector can be derived $\infty$ times. Let's first proof that the planar clothoid (Eq. (9)) is $C^{\infty}$. Its $n$-th derivative is continuous and differentiable, and can be expressed as

$$
\mathbf{C}^{(n)}(s, \mathbf{p})=\left[\begin{array}{c}
\tilde{c}_{1, n}(s, \mathbf{p}) \sin (\beta(s, \mathbf{p}))+\tilde{c}_{2, n}(s, \mathbf{p}) \cos (\beta(s, \mathbf{p})) \\
\bar{c}_{1, n}(s, \mathbf{p}) \sin (\beta(s, \mathbf{p}))+\bar{c}_{2, n}(s, \mathbf{p}) \cos (\beta(s, \mathbf{p}))
\end{array}\right]
$$

for some polynomials $\tilde{c}_{1, n}(s, \mathbf{p}), \tilde{c}_{2, n}(s, \mathbf{p}), \bar{c}_{1, n}(s, \mathbf{p}), \bar{c}_{2, n}(s, \mathbf{p})$.

It is then clear that the $n$-th derivative of Eq. (12) can be expressed as

$$
\mathbf{C}^{(n)}\left(s, \mathbf{p}_{1}, \mathbf{p}_{2}\right)=\left[\begin{array}{l}
\tilde{d}_{1, n}(\star) \sin (\psi(\bullet))+\tilde{d}_{2, n}(\star) \cos (\psi(\bullet)) \\
\bar{d}_{1, n}(\star) \sin (\psi(\bullet))+\bar{d}_{2, n}(\star) \cos (\psi(\bullet)) \\
\hat{d}_{1, n}(\bullet) \sin (\theta(\bullet))+\hat{d}_{2, n}(\bullet) \cos (\theta(\bullet))
\end{array}\right]
$$

where $\tilde{d}_{1, n}(\star), \tilde{d}_{2, n}(\star), \bar{d}_{1, n}(\star)$, and $\bar{d}_{2, n}(\star)$ denote some polynomials and trigonometric expressions related to $\theta(\bullet)$ and its derivatives (which are also continuous and differentiable); while $\hat{d}_{1, n}(\bullet)$ and $\hat{d}_{2, n}(\bullet)$ are some polynomials of $s$, as long as $\epsilon_{0} \neq 0$ or $\mu \neq 0 ; v_{0} \neq 0$ or $\rho \neq 0$. Details omitted for brevity.

D Orientation From fundamental theorem of calculus and using the chain rule, we get the following equality

$$
\frac{d}{d x} \int_{a(x)}^{b(x)} f(t) d t=f(b(x)) b^{\prime}(x)-f(a(x)) a^{\prime}(x)
$$

being $f(t)$ an integrable function over the range $[a(x), b(x)]$, with $a(x)$ and $b(x)$ derivable functions. 
From Eq. [12] a Cb3D curve can be expressed explicitly as

$$
\mathbf{C}\left(s, \mathbf{p}_{1}, \mathbf{p}_{2}\right):=\left\{\begin{array}{l}
\int_{0}^{C\left(s, \mathbf{p}_{2}\right)} \cos \left(\psi_{0}+\epsilon_{0} \xi+\frac{\mu}{2} \xi^{2}\right) d \xi \\
\int_{0}^{C\left(s, \mathbf{p}_{2}\right)} \sin \left(\psi_{0}+\epsilon_{0} \xi+\frac{\mu}{2} \xi^{2}\right) d \xi \\
-\int_{0}^{s} \sin \left(\theta_{0}+v_{0} \xi+\frac{\rho}{2} \xi^{2}\right) d \xi
\end{array}\right.
$$

with

$$
C\left(s, \mathbf{p}_{2}\right):=\int_{0}^{s} \cos \left(\theta_{0}+v_{0} \xi+\frac{\rho}{2} \xi^{2}\right) d \xi
$$

Hence, from Eqs. (3) and (12) the tangent vector can be derived from position vector, such that

$$
\mathbf{C}^{\prime}\left(s, \mathbf{p}_{1}, \mathbf{p}_{2}\right):=\left\{\begin{array}{l}
\cos \left(\psi_{0}+\epsilon_{0} C\left(s, \mathbf{p}_{2}\right)+\frac{\mu}{2} C^{2}\left(s, \mathbf{p}_{2}\right)\right) C^{\prime}\left(s, \mathbf{p}_{2}\right) \\
\sin \left(\psi_{0}+\epsilon_{0} C\left(s, \mathbf{p}_{2}\right)+\frac{\mu}{2} C^{2}\left(s, \mathbf{p}_{2}\right)\right) C^{\prime}\left(s, \mathbf{p}_{2}\right) \\
-\sin \left(\theta_{0}+v_{0} s+\frac{\rho}{2} s^{2}\right)
\end{array}\right.
$$

where

$$
C^{\prime}\left(s, \mathbf{p}_{2}\right):=\cos \left(\theta_{0}+v_{0} s+\frac{\rho}{2} s^{2}\right)
$$

On the other hand, for any space curve in $\mathbb{R}^{3}$, orientation can be defined in $\mathcal{S}^{3}$ by Euler angles roll $\phi(s)$, pitch $\theta(s)$, and yaw $\psi(s)$. Using algebra of rotation matrices, orientation can be expressed as a product of rotation matrices so that

$$
\mathbf{R}(\phi(s), \theta(s), \psi(s)):=\mathbf{R}_{\mathbf{z}}(\psi(s)) \mathbf{R}_{\mathbf{y}}(\theta(s)) \mathbf{R}_{\mathbf{x}}(\phi(s))
$$

being $\mathbf{R}_{\mathbf{x}}(\bullet), \mathbf{R}_{\mathbf{y}}(\bullet)$, and $\mathbf{R}_{\mathbf{z}}(\bullet)$ three-dimensional rotation matrices around each axis of the global frame.

The first column of a rotation matrix represents the tangent vector $\mathbf{T}(s)$, computed as in Eq. [16, whose components are defined with respect to a global coordinate system.

Finally, the module of Eq. (43) can be computed easily to get $\left|\mathbf{C}^{\prime}\left(s, \mathbf{p}_{1}, \mathbf{p}_{2}\right)\right|=1$ and, as a consequence, Eq. (3) becomes $\mathbf{T}\left(s, \mathbf{p}_{1}, \mathbf{p}_{2}\right)=\mathbf{C}^{\prime}\left(s, \mathbf{p}_{1}, \mathbf{p}_{2}\right)$. Therefore, from Eqs. (43) and (44) it is easy to proof the definitions of Eqs. (13) and 14. 
E Straight line A straight line in $\mathbb{R}^{3}$ is defined as

$$
\mathbf{C}(s, \mathbf{p}):=\left\{\begin{array}{c}
x(s, \mathbf{p})=a s \\
y(s, \mathbf{p})=b s \\
z(s, \mathbf{p})=c s
\end{array}\right.
$$

where $a, b$, and $c$ are constants.

For $\mathbf{p}_{1}=\left\{\forall \psi_{0}, \epsilon_{0}=0, \mu=0\right\}$ and $\mathbf{p}_{2}=\left\{\forall \theta_{0}, v_{0}=0, \rho=0\right\}$ it is straightforward to see that Eq. (12), expressed explicitly in Eqs. 41] and (42, becomes

$$
\begin{aligned}
\mathcal{C}\left(s, \mathbf{p}_{2}\right) & =\int_{0}^{s} \cos \left(\theta_{0}\right) d \xi=\cos \left(\theta_{0}\right) s \\
-\mathcal{S}\left(s, \mathbf{p}_{2}\right) & =-\int_{0}^{s} \sin \left(\theta_{0}\right) d \xi=-\sin \left(\theta_{0}\right) s \\
\mathcal{C}\left(C\left(s, \mathbf{p}_{2}\right), \mathbf{p}_{1}\right) & =\int_{0}^{\cos \left(\theta_{0}\right) s} \cos \left(\psi_{0}\right) d \xi=\cos \left(\psi_{0}\right) \cos \left(\theta_{0}\right) s \\
\mathcal{S}\left(C\left(s, \mathbf{p}_{2}\right), \mathbf{p}_{1}\right) & =\int_{0}^{\cos \left(\theta_{0}\right) s} \sin \left(\psi_{0}\right) d \xi=\sin \left(\psi_{0}\right) \cos \left(\theta_{0}\right) s
\end{aligned}
$$

Thus, from Eqs. 46, (48), 49], and (50) we can state $a=\cos \left(\psi_{0}\right) \cos \left(\theta_{0}\right), b=\sin \left(\psi_{0}\right) \cos \left(\theta_{0}\right)$, and $c=-\sin \left(\theta_{0}\right)$.

F Circular arc A circle in $\mathbb{R}^{3}$ can be defined as

$$
\mathbf{C}(s, \mathbf{p}):=\left\{\begin{array}{c}
x(s, \mathbf{p})=a \cos (b+c s) \\
y(s, \mathbf{p})=a \sin (b+c s) \\
z(s, \mathbf{p})=d
\end{array}\right.
$$

being $a$ the radius, $b$ an initial angular offset, $c s$ the turning angle traveled by the circle and $d$ a plane parallel to $z=0$.

For $\mathbf{p}_{1}=\left\{\forall \psi_{0}, \epsilon_{0} \neq 0, \mu=0\right\}$ and $\mathbf{p}_{2}=\mathbf{0}$ we can see that Eqs. (41) and (42) become

$$
\begin{aligned}
C\left(s, \mathbf{p}_{2}\right) & =\int_{0}^{s} \cos (0) d \xi=s \\
-\mathcal{S}\left(s, \mathbf{p}_{2}\right) & =-\int_{0}^{s} \sin (0) d \xi=0 \\
\mathcal{C}\left(C\left(s, \mathbf{p}_{2}\right), \mathbf{p}_{1}\right) & =\int_{0}^{s} \cos \left(\psi_{0}+\epsilon_{0} \xi\right) d \xi=\frac{\sin \left(\psi_{0}+\epsilon_{0} s\right)}{\epsilon_{0}} \\
\mathcal{S}\left(C\left(s, \mathbf{p}_{2}\right), \mathbf{p}_{1}\right) & =\int_{0}^{s} \sin \left(\psi_{0}+\epsilon_{0} \xi\right) d \xi=\frac{-\cos \left(\psi_{0}+\epsilon_{0} s\right)}{\epsilon_{0}}
\end{aligned}
$$


From trigonometric relationships $\cos (w)=\sin (\pi / 2-w), \sin (w)=\cos (\pi / 2-w), \cos (-w)=\cos (w)$ and $\sin (-w)=-\sin (w)$, Eqs. (54) and (55) can be rewritten as

$$
\begin{aligned}
& C\left(C\left(s, \mathbf{p}_{2}\right), \mathbf{p}_{1}\right)=\frac{\cos \left(\psi_{0}-\pi / 2+\epsilon_{0} s\right)}{\epsilon_{0}} \\
& \mathcal{S}\left(C\left(s, \mathbf{p}_{2}\right), \mathbf{p}_{1}\right)=\frac{\sin \left(\psi_{0}-\pi / 2+\epsilon_{0} s\right)}{\epsilon_{0}}
\end{aligned}
$$

Then, from Eqs. (51), 53, 56, and (57) we get the following equalities $a=1 / \epsilon_{0}, b=\psi_{0}-\pi / 2, c=\epsilon_{0}$, and $d=0$.

Proof for the case of $\mathbf{p}_{1}=\left\{\forall \psi_{0}, \epsilon_{0}=0, \mu=0\right\}$ and $\mathbf{p}_{2}=\left\{\forall \theta_{0}, v_{0} \neq 0, \rho=0\right\}$ is omitted for brevity, although it can be easily obtained.

G Planar clothoid A planar clothoid in $\mathbb{R}^{2}$ is defined as in Eq. (9). But, for a planar clothoid contained in the $X Z$ plane, equations can be expressed in $\mathbb{R}^{3}$ as follows

$$
\mathbf{C}(s, \mathbf{p}):=\left\{\begin{array}{l}
x(s, \mathbf{p})=C(s, \mathbf{p}) \\
y(s, \mathbf{p})=0 \\
z(s, \mathbf{p})=-\mathcal{S}(s, \mathbf{p})
\end{array}\right.
$$

For $\mathbf{p}_{1}=\mathbf{0}$ and $\forall \mathbf{p}_{2}$, Eqs. (41) and 42) can be rewritten as

$$
\begin{aligned}
C\left(s, \mathbf{p}_{2}\right) & =\int_{0}^{s} \cos \left(\theta_{0}+v_{0} \xi+\frac{\rho}{2} \xi^{2}\right) d \xi \\
-\mathcal{S}\left(s, \mathbf{p}_{2}\right) & =-\int_{0}^{s} \sin \left(\theta_{0}+v_{0} \xi+\frac{\rho}{2} \xi^{)} d \xi\right. \\
C\left(C\left(s, \mathbf{p}_{2}\right), \mathbf{p}_{1}\right) & =\int_{0}^{C\left(s, \mathbf{p}_{2}\right)} \cos (0) d \xi=C\left(s, \mathbf{p}_{2}\right) \\
\mathcal{S}\left(C\left(s, \mathbf{p}_{2}\right), \mathbf{p}_{1}\right) & =\int_{0}^{C\left(s, \mathbf{p}_{2}\right)} \sin (0) d \xi=0
\end{aligned}
$$

Therefore, it is straightforward to see that Eqs. 60, 61, and 62 compose a 2D clothoid contained in the $X Z$ plane.

Proof for the case of $\mathbf{p}_{2}=\mathbf{0}$ and $\forall \mathbf{p}_{1}$ is omitted for brevity, although it can be easily obtained following the same argumentation as in proof of circular arc's property. 
H Circular helix A circular helix of radius $a$ and slope $d / a$ can be parametrized by arc-length $s$ as follows

$$
\mathbf{C}(s, \mathbf{p}):=\left\{\begin{array}{l}
x(s, \mathbf{p})=a \cos (b+c s) \\
y(s, \mathbf{p})=a \sin (b+c s) \\
z(s, \mathbf{p})=d c s
\end{array}\right.
$$

with $b$ an initial angular offset, $c=1 / \sqrt{a^{2}+d^{2}}$, and $c s$ the turning angle traveled by the helix.

For $\mathbf{p}_{1}=\left\{\forall \psi_{0}, \epsilon_{0} \neq 0, \mu=0\right\}$ and $\mathbf{p}_{2}=\left\{\forall \theta_{0}, v_{0}=0, \rho=0\right\}$ it is straightforward to see that Eqs. (41) and (42) can be expressed as

$$
\begin{aligned}
& C\left(s, \mathbf{p}_{2}\right)=\int_{0}^{s} \cos \left(\theta_{0}\right) d \xi=\cos \left(\theta_{0}\right) s \\
& -\mathcal{S}\left(s, \mathbf{p}_{2}\right)=-\int_{0}^{s} \sin \left(\theta_{0}\right) d \xi=-\sin \left(\theta_{0}\right) s \\
& \mathcal{C}\left(C\left(s, \mathbf{p}_{2}\right), \mathbf{p}_{1}\right)=\int_{0}^{\cos \left(\theta_{0}\right) s} \cos \left(\psi_{0}+\epsilon_{0} \xi\right) d \xi= \\
& \mathcal{S}\left(C\left(s, \mathbf{p}_{2}\right), \mathbf{p}_{1}\right)=\int_{0}^{\cos \left(\theta_{0}\right) s} \sin \left(\psi_{0}+\epsilon_{0} \cos \left(\theta_{0}\right) s\right) \\
& \epsilon_{0} \\
& =\frac{-\cos \left(\psi_{0}+\epsilon_{0} \cos \left(\theta_{0}\right) s\right)}{\epsilon_{0}}
\end{aligned}
$$

From trigonometric relationships, Eqs. 66 and (67) can be rewritten as

$$
\begin{aligned}
& C\left(C\left(s, \mathbf{p}_{2}\right), \mathbf{p}_{1}\right)=\frac{\cos \left(\psi_{0}-\pi / 2+\epsilon_{0} \cos \left(\theta_{0}\right) s\right)}{\epsilon_{0}} \\
& \mathcal{S}\left(C\left(s, \mathbf{p}_{2}\right), \mathbf{p}_{1}\right)=\frac{\sin \left(\psi_{0}-\pi / 2+\epsilon_{0} \cos \left(\theta_{0}\right) s\right)}{\epsilon_{0}}
\end{aligned}
$$

Finally, from Eqs. 63, 65, 68, and 69) we get the following equalities $a=1 / \epsilon_{0}, b=\psi_{0}-\pi / 2, c=\epsilon_{0} \cos \left(\theta_{0}\right)$, and $d=-\sin \left(\theta_{0}\right) / c=-\tan \left(\theta_{0}\right) / \epsilon_{0}$.

I Monotonic increasing position Cb3D curves can be defined in all 8 octants using symmetries (see Fig. 3 . Depending on each case the elements of position vector will be either monotonic increasing or decreasing. A monotonic decreasing function become monotonic increasing when using the absolute value. In this sense, using position vector's absolute value, all 8 cases can be reduced to only one. When $\mathbf{C}\left(s, \mathbf{p}_{1}, \mathbf{p}_{2}\right)=\left|\mathbf{C}\left(s, \mathbf{p}_{1}, \mathbf{p}_{2}\right)\right|$ the final angles are $\theta\left(s, \mathbf{p}_{2}\right) \in[-\pi / 2,0]$ and $\psi\left(s, \mathbf{p}_{1}, \mathbf{p}_{2}\right) \in[0, \pi / 2]$, with $s>0$. Hence, under these conditions the position vector 
$\mathbf{C}\left(s, \mathbf{p}_{1}, \mathbf{p}_{2}\right)$ of a Cb3D curve is monotonically increasing, iff

$$
\mathbf{C}^{\prime}\left(s, \mathbf{p}_{1}, \mathbf{p}_{2}\right):=\left\{\begin{array}{r}
\cos \psi\left(s, \mathbf{p}_{1}, \mathbf{p}_{2}\right) \cos \theta\left(s, \mathbf{p}_{2}\right) \geq 0 \\
\sin \psi\left(s, \mathbf{p}_{1}, \mathbf{p}_{2}\right) \cos \theta\left(s, \mathbf{p}_{2}\right) \geq 0 \\
-\sin \theta\left(s, \mathbf{p}_{2}\right) \geq 0
\end{array}\right.
$$

For equations in Eq. (70) to be true, the following conditions need to be true

$$
\begin{aligned}
& \cos \psi\left(s, \mathbf{p}_{1}, \mathbf{p}_{2}\right) \geq 0, \quad \cos \theta\left(s, \mathbf{p}_{2}\right) \geq 0 \\
& \sin \psi\left(s, \mathbf{p}_{1}, \mathbf{p}_{2}\right) \geq 0,-\sin \theta\left(s, \mathbf{p}_{2}\right) \geq 0
\end{aligned}
$$

which are true in the defined domain $\theta\left(s, \mathbf{p}_{2}\right) \in[-\pi / 2,0]$ and $\psi\left(s, \mathbf{p}_{1}, \mathbf{p}_{2}\right) \in[0, \pi / 2]$, iff $-\pi / 2 \leq \theta_{0} \leq 0,0 \leq \psi_{0} \leq \pi / 2$, $v_{0} \leq 0, \epsilon_{0} \geq 0, \rho \leq 0$, and $\mu \geq 0$.

J Monotonic increasing orientation As in the previous property, depending on each case the angles $\theta\left(s, \mathbf{p}_{2}\right)$ and $\psi\left(s, \mathbf{p}_{1}, \mathbf{p}_{2}\right)$ can be either monotonically increasing or decreasing. However, all 8 cases can be reduced to only one, where $\theta\left(s, \mathbf{p}_{2}\right)=\left|\theta\left(s, \mathbf{p}_{2}\right)\right|$ and $\psi\left(s, \mathbf{p}_{1}, \mathbf{p}_{2}\right)=\left|\psi\left(s, \mathbf{p}_{1}, \mathbf{p}_{2}\right)\right|$. So, without loss of generality we only focus in such case to proof that the statement is true for $\theta\left(s, \mathbf{p}_{2}\right) \in[0, \pi / 2]$ and $\psi\left(s, \mathbf{p}_{1}, \mathbf{p}_{2}\right) \in[0, \pi]$, with $s>0$.

For angles yaw and pitch to be monotonic increasing, the following conditions in the derivatives of Eqs. 13 and (14) must be satisfied

$$
\begin{aligned}
\theta^{\prime}\left(s, \mathbf{p}_{2}\right) & =v_{0}+\rho s \geq 0 \\
\psi^{\prime}\left(s, \mathbf{p}_{1}, \mathbf{p}_{2}\right) & =\epsilon_{0}+\mu C\left(s, \mathbf{p}_{2}\right) C^{\prime}\left(s, \mathbf{p}_{2}\right) \geq 0
\end{aligned}
$$

For Eqs. (72) and (73) to be true, the following conditions need to be true

$$
\begin{aligned}
& v_{0} \geq 0, \quad \rho \geq 0, \\
& \epsilon_{0} \geq 0, \quad \mu \geq 0, \quad C\left(s, \mathbf{p}_{2}\right) \geq 0, \quad C^{\prime}\left(s, \mathbf{p}_{2}\right) \geq 0 .
\end{aligned}
$$

which are all true in the defined range for $s>0$, iff $C^{\prime}\left(s, \mathbf{p}_{2}\right)=\cos \left(\theta_{0}+v_{0} s+\frac{\rho}{2} s^{2}\right) \geq 0$, that is only true if $0 \leq \theta_{0} \leq \pi / 2$ since $\theta\left(s, \mathbf{p}_{2}\right) \in[0, \pi / 2]$. Also considering that $\psi\left(s, \mathbf{p}_{1}, \mathbf{p}_{2}\right) \in[0, \pi]$, the condition $0 \leq \psi_{0} \leq \pi$ must be also required. 


\section{Funding Sources}

This work was supported by Generalitat Valenciana under the postdoctoral grant APOSTD/2017/055. The authors are also grateful to the financial support of Spanish Ministry of Economy and European Union, grant DPI2016-81002-R (AEI/FEDER, UE).

\section{References}

[1] C. Goerzen, Z. K., Mettler, B., Goerzen, C., Kong, Z., and Mettler, B., “A Survey of Motion Planning Algorithms from the Perspective of Autonomous UAV Guidance,” Journal of Intelligent Robots and Systems, Vol. 57, No. 1-4, 2010, pp. 65-100. doi:10.1007/s10846-009-9383-1.

[2] Sujit, P., Saripalli, S., and Sousa, J. B., "Unmanned Aerial Vehicle Path Following: A Survey and Analysis of Algorithms for FixedWing Unmanned Aerial Vehicless,” IEEE Control Systems, Vol. 34, No. 1, 2014, pp. 42-59. doi:10.1109/MCS.2013.2287568.

[3] Zeng, Z., Lian, L., Sammut, K., He, F., Tang, Y., and Lammas, A., “A survey on path planning for persistent autonomy of autonomous underwater vehicles," Ocean Engineering, Vol. 110, 2015, pp. 303-313. doi:10.1016/j.oceaneng.2015.10.007.

[4] Adiprawita, W., Suwandi Ahmad, A., and Sembiring, J., "Development of ahrs (attitude and heading reference system) for autonomous uav (unmanned aerial vehicle)," 2012.

[5] Zhai, R., Zhou, Z., Zhang, W., Sang, S., and Li, P., "Control and navigation system for a fixed-wing unmanned aerial vehicle," AIP Advances, Vol. 4, No. 3, 2014, p. 031306. doi:10.1063/1.4866169.

[6] Rubio Hervas, J., Reyhanoglu, M., Tang, H., and Kayacan, E., "Nonlinear control of fixed-wing UAVs in presence of stochastic winds," Communications in Nonlinear Science and Numerical Simulation, Vol. 33, 2016, pp. 57-69. doi: 10.1016/J.CNSNS.2015.08.026.

[7] Bhandari, S., Lu, Y., Raheja, A., and Tang, D., "Nonlinear Control of a Fixed-Wing UAV using Support Vector Machine," AIAA Guidance, Navigation, and Control Conference, American Institute of Aeronautics and Astronautics, Reston, Virginia, 2016. doi:10.2514/6.2016-0107.

[8] Nguen, V. F., Putov, A. V., and Nguen, T. T., “Adaptive control of an unmanned aerial vehicle," AIP Conference Proceedings, Vol. 1798, AIP Publishing LLC, 2017, p. 020124. doi:10.1063/1.4972716.

[9] Prabhakar, N., Painter, A., Prazenica, R., and Balas, M., “Trajectory-Driven Adaptive Control of Autonomous Unmanned Aerial Vehicles with Disturbance Accommodation," Journal of Guidance, Control, and Dynamics, Vol. 41, No. 9, 2018 , pp. 1976-1989. doi:10.2514/1.G003341.

[10] Eren, U., Prach, A., Koçer, B. B., Raković, S. V., Kayacan, E., and Açıkmeşe, B., "Model Predictive Control in Aerospace Systems: Current State and Opportunities,” Journal of Guidance, Control, and Dynamics, Vol. 40, No. 7, 2017, pp. 1541-1566. doi:10.2514/1.G002507. 
[11] Liu, M., Liu, H., and Wei, X., "Autonomous position holding and point to point flying control of quadrotor UAVs," Proceeding of the 11th World Congress on Intelligent Control and Automation, IEEE, 2014, pp. 2389-2394. doi:10.1109/wcica.2014.7053095.

[12] Liang, H., Zhong, W., and Chunhui, Z., "Point-to-point near-optimal obstacle avoidance path for the unmanned aerial vehicle," 2015 34th Chinese Control Conference (CCC), IEEE, 2015, pp. 5413-5418. doi:10.1109/chicc.2015.7260486.

[13] Kan, E.-M., Lim, M.-H., Yeo, S.-P., Ho, J.-S., and Shao, Z., "Contour Based Path Planning with B-Spline Trajectory Generation for Unmanned Aerial Vehicles (UAVs) over Hostile Terrain,” Journal of Intelligent Learning Systems and Applications, Vol. 03, No. 03, 2011, pp. 122-130. doi:10.4236/jilsa.2011.33014.

[14] Jung, D., and Tsiotras, P., “On-Line Path Generation for Unmanned Aerial Vehicles Using B-Spline Path Templates,” Journal of Guidance, Control, and Dynamics, Vol. 36, No. 6, 2013, pp. 1642-1653. doi:10.2514/1.60780.

[15] Choe, R., Puig-Navarro, J., Cichella, V., Xargay, E., and Hovakimyan, N., "Cooperative Trajectory Generation Using Pythagorean Hodograph Bézier Curves,” Journal of Guidance, Control, and Dynamics, Vol. 39, No. 8, 2016 , pp. 1744-1763. doi:10.2514/1.G001531.

[16] de Dilectis, F., Mortari, D., and Zanetti, R., "Bézier Description of Space Trajectories,” Journal of Guidance, Control, and Dynamics, Vol. 39, No. 11, 2016, pp. 2535-2539. doi:10.2514/1.G000719.

[17] Wang, X., Jiang, P., Li, D., and Sun, T., “Curvature continuous and bounded path planning for fixed-wing UAVs," Sensors (Switzerland), Vol. 17, No. 9, 2017, pp. 1-21. doi:10.3390/s17092155.

[18] Pérez, L. H., Aguilar, M. C. M., Sánchez, N. M., and Montesinos, A. F., "Path Planning Based on Parametric Curves," Advanced Path Planning for Mobile Entities, InTech, 2018. doi:10.5772/intechopen.72574.

[19] Mittal, S., and Deb, K., "Three-Dimentional Offline Path Planning for UAVs Using Multiobjective Evolutionary Algorithms," 2007 IEEE Congress on Evolutionary Computation (CEC'2007), , No. i, 2007, pp. 3195-3202. doi:10.1109/CEC.2007.4424880.

[20] Hota, S., and Ghose, D., "Optimal path planning for an aerial vehicle in 3D space," 49th IEEE Conference on Decision and Control (CDC), 2010, pp. 4902-4907. doi:10.1109/CDC.2010.5717246.

[21] Rikovitch, N., and Sharf, I., "Kinodynamic Motion Planning for UAVs: A Minimum Energy Approach," AIAA Guidance, Navigation, and Control (GNC) Conference, American Institute of Aeronautics and Astronautics, Reston, Virginia, 2013. doi:10.2514/6.2013-5231.

[22] Ataei, M., and Yousefi-Koma, A., "Three-dimensional optimal path planning for waypoint guidance of an autonomous underwater vehicle," Robotics and Autonomous Systems, Vol. 67, 2015, pp. 23-32. doi:10.1016/j.robot.2014.10.007.

[23] Gonzalez, D., Perez, J., Milanes, V., and Nashashibi, F., “A Review of Motion Planning Techniques for Automated Vehicles,” IEEE Transactions on Intelligent Transportation Systems, Vol. 17, No. 4, 2016, pp. 1135-1145. doi:10.1109/TITS.2015.2498841.

[24] Shin, D. H., and Singh, S., "Path Generation for Robot Vehicles Using Composite Clothoid Segments," Tech. Rep. CMU-RITR-90-31, Robotics Institute, Pittsburgh, PA, 1990. 
[25] Delingette, H., Hebert, M., and Lkeuchi, K., "Trajectory Generation with Curvature Constraint based on Energy Minimization," IEEE Int. Conf. on Intelligent Robots and Systems, 1991.

[26] Tounsi, M., and Le Corre, J. F., "Trajectory generation for mobile robots," Mathematics and Computers in Simulation, Vol. 41, No. 3-4, 1996, pp. 367-376. doi:http://dx.doi.org/10.1016/0378-4754(95)00085-2.

[27] amd Alonzo Kelly, B. N., “Trajectory Generation for Car-Like Robots Using Cubic Curvature Polynomials," Field and Service Robots, 2001.

[28] Levien, R., "Technical Report UCB/EECS-2008-111, The Euler spiral: a mathematical history,” Tech. rep., EECS Department, University of California, Berkeley, 2008.

[29] Fraichard, T., and Scheuer, A., "From Reeds and Shepp's to Continuous-Curvature Paths," Robotics and Automation, IEEE Transactions on, Vol. 20, 2004, pp. 1025-1035. doi:10.1109/tro.2004.833789.

[30] Girbés, V., Armesto, L., and Tornero, J., "Path Following Hybrid Control for Vehicle Stability Applied to Industrial Forklifts," Robotics and Autonomous Systems, Vol. 62, No. 6, 2014, pp. 910-922. doi:10.1016/j.robot.2014.01.004.

[31] Armesto, L., Girbés, V., Vincze, M., Olufs, S., and Muñoz-Benavent, P., "Mobile Robot Obstacle Avoidance Based on Quasi-Holonomic Smooth Paths,” Advances in Autonomous Robotics, Vol. 7429 LNAI, Springer Berlin Heidelberg, 2012, pp. 244-255. doi:10.1007/978-3-642-32527-4_22.

[32] Brezak, M., and Petrovic, I., "Real-time Approximation of Clothoids With Bounded Error for Path Planning Applications," IEEE Transactions on Robotics, Vol. 30, No. 2, 2014, pp. 507-515. doi:10.1109/tro.2013.2283928.

[33] Yang, K., and Sukkarieh, S., "3D smooth path planning for a UAV in cluttered natural environments," 2008 IEEE/RSJ International Conference on Intelligent Robots and Systems, IROS, 2008, pp. 794-800. doi:10.1109/IROS.2008.4650637.

[34] Wan, T. R., Tang, W., Chen, H., Tao Ruan Wan, W. T., and Chen, H., "A real-time 3D motion planning and simulation scheme for nonholonomic systems," Simulation Modelling Practice and Theory, Vol. 19, No. 1, 2011, pp. 423-439. doi: 10.1016/j.simpat.2010.08.002.

[35] Wilburn, J. N., Perhinschi, M. G., and Wilburn, B. K., "Implementation of Composite Clothoid Paths for Continuous Curvature Trajectory Generation for UAVs," AIAA Guidance, Navigation, and Control (GNC) Conference, American Institute of Aeronautics and Astronautics, Reston, Virginia, 2013. doi:10.2514/6.2013-5230.

[36] Kommer, A., and Weidner, T., "Some aspects of clothoids," Proceedings of 7th International Conference on Applied Informatics, Vol. 1, 2007, pp. 115-120.

[37] Harary, G., and Tal, A., "3D Euler spirals for 3D curve completion," Computational Geometry: Theory and Applications, Vol. 45, No. 3, 2012, pp. 115-126. doi:10.1016/j.comgeo.2011.10.001.

[38] Bertails-Descoubes, F., "Super-clothoids," Computer Graphics Forum, Vol. 31, No. 2, 2012, pp. 509-518. doi:10.1111/j.14678659.2012.03030.x. 
[39] Çelik, Semra S.; Yayli, Yusuf; Güler, E., "On Generalized Euler Spirals in E3,” Differential Geometry, Vol. 5, No. 1, 2016, pp. 5-14.

[40] Banchoff, T., and Lovett, S. S. T., Differential geometry of curves and surfaces, A K Peters/CRC Press, 2010. doi: $10.1201 / 9781439894057$.

[41] Meek, D. S., and Walton, D. J., “A note on finding clothoids,” Journal of Computational and Applied Mathematics, Vol. 171, No. 1-2, 2004, pp. 433-453. doi:10.1016/j.cam.2003.12.047.

[42] Marzbani, H., Jazar, R. N., and Fard, M., "Better Road Design Using Clothoids," Sustainable Automotive Technologies 2014, Springer International Publishing, 2015, pp. 25-40. doi:10.1007/978-3-319-17999-5_3.

[43] Marzbani, H., Simic, M., Fard, M., and Jazar, R. N., "Better Road Design for Autonomous Vehicles Using Clothoids," Intelligent Interactive Multimedia Systems and Services, Springer International Publishing, 2015, pp. 265-278. doi:10.1007/978-3-31919830-9_24.

[44] Gim, S., Adouane, L., Lee, S., and Dérutin, J. P., "Clothoids Composition Method for Smooth Path Generation of Car-Like Vehicle Navigation,” Journal of Intelligent and Robotic Systems: Theory and Applications, Vol. 88, No. 1, 2017, pp. 129-146. doi:10.1007/s10846-017-0531-8.

[45] Mielenz, K. D., “Computation of Fresnel Integrals II,” Journal of Research of the National Institute of Standards and Technology, Vol. 105, No. 4, 2000, pp. 589-590. doi:10.6028/jres.105.049.

[46] Montés, N., Herraez, A., Armesto, L., and Tornero, J., "Real-time clothoid approximation by Rational Bezier curves," IEEE International Conference on Robotics and Automation, 2008, pp. 2246-2251. doi:10.1109/ROBOT.2008.4543548.

[47] Narayan, S., “Approximating Cornu spirals by arc splines,” Journal of Computational and Applied Mathematics, Vol. 255, 2014, pp. 789-804. doi:10.1016/j.cam.2013.06.038.

[48] Chen, Y., Cai, Y., Zheng, J., and Thalmann, D., "Accurate and Efficient Approximation of Clothoids Using Bézier Curves for Path Planning,” IEEE Transactions on Robotics, Vol. 33, No. 5, 2017, pp. 1242-1247. doi:10.1109/TRO.2017.2699670.

[49] Press, William H., Teukolsky, Saul A., Vetterling, William T., and Flannery, B. P., Numerical recipes: the art of scientific computing, $3^{\text {rd }}$ ed., Cambridge University Press, 2007.

[50] Knuth, D. E., "Mathematical typography," Bulletin of the American Mathematical Society, Vol. 1, No. 2, 1979 , pp. $337-372$. doi:10.1090/s0273-0979-1979-14598-1.

[51] Kostov, V., and Degtiariova-Kostova, E., “Some Properties of Clothoids,” Tech. rep., RR-2752, INRIA, 1995.

[52] Velasco-Carrau, J., García-Nieto, S., Salcedo, J., and Bishop, R. H., "Multi-objective optimization for wind estimation and aircraft model identification,” Journal of Guidance, Control, and Dynamics, Vol. 39, No. 2, 2015, pp. 372-389. 